\title{
Bath and the body, bamboo and the soul
}

Thesis is submitted to the facility of Architecture and Design Wellington in partial fulfilment of the requirements for the degree of Master of Architecture (professional). Victoria University of Wellington.

December $14^{\text {th }}, 2010$

Keywords: Bath ritual, Bathing, Bamboo, Bamboo preservation Industrial bamboo, New Zealand bamboo.

Dion Palamountain 
Page | 2 


\section{Abstract}

Bathing at a fundamental level is desired for two attributes cleansing the body, and cleansing the soul. Historically the act of bathing has been a combination of hot and cold water bathing; this act cleanses both the body and soul, and is seen as an enjoyable pastime for both eastern and western cultures. The priority then is to architecturally express the 'body and soul' through a careful material selection. The choice of materials reflects this, concrete and bamboo, expressing the solid (body) and the light (soul).

Design precedents, material comparison, eastern and western beliefs are used to establish a rationale between the material contrast of body and soul. The location for the design reflects the natural connection between 'body and soul' in a location that references the land and water, geothermal and steam, lake and landscape.

The final project includes detailing of non-penetrating fixings for the use in bamboo construction, including natural and industrial products into a public bath that compliments the body and the soul. 
Page 4 


\section{Introduction}

2. Bathing and the body

2.1 Greek baths

2.2 Roman baths

2.3 Medieval baths

2.4 Japanese baths

2.5 Summary

3. Bath and stone

4. Town, stone and water

4.1 Site and water

5. The material of the soul

5. The soul of bamboo

6. The material bamboo

6.1 Material characteristics

6.2 Durability

6.3 Industrial bamboo materials

6.3.1 Bamboo lumber

6.3.1 Bamboo matboard

6.4 Summary

7. Design

7.1 Bamboo design

7.1.1 Bamboo concept

7.1.2 Concept discussion

7.1.3 New Zealand timber bamboo

7.1.4 Harvesting and processing

7.1.5 Experimentation

7.1.6 Experimentation assessment
7.1.7 Bamboo development

7.1.8 Development discussion

7.1.9 Bamboo Summary

7.2 Design solution

7.2.1 Design rationale

7.2.2 Bathing and the body

7.2.3 Bath and stone

7.2.4 Site and water

7.2.5 Bamboo and soul

7.2.6 Structure and bamboo

\section{Design discussion}

p. 71

10. Conclusion

p. 73 
Page 6 


\section{Introduction}

\section{Problem}

There is a fundamental connection between the body and the bath. This is commonly seen in how historic bathing is addressed in terms of the ritual. However at its most primal, the bath is seen as not just a connection between the body, but also the soul. How then can materiality in architecture connect the body and the soul, in a thermal bath?

Aim

- To design a set of public thermal baths that benefits the sensory perceptions, of both body and soul, through materiality.

- To design a contemporary bathhouse in the New Zealand context that incorporates historic bathing rituals.
- To find a material that expresses and respects the ontology of a contemporary bath.

\section{Approach}

There are two distinct ideas interacting in this research, that of the body and bath, and that of the body and soul. The research will be undertaken in relation to these two principles by firstly identifying the ritual of the bath as it connects the body to the bath, and secondly using the material bamboo to reflect the connection between the body and the soul.

In order to understand the act of bathing and it relationship to the body, research into historic bating rituals will be undertaken. Having knowledge of the bathing process will help define the spatial planning of the design, by looking at the typologies of bathhouses in defining a layout. The connection between relaxation and recreation will also be identified to further illustrate the connection between the body and bathing as it is important in connecting it to a material association. My decision to carry out this research was influenced by Peter 
Zumthor's Therme Vals and his approach to the building's architecture, material and mass in relationship to the body.

The location of my project in the geothermal landscape of Taupo allows for a connection to the natural environment that reflects the requirements of a thermal bath. The choice of the selected site has been guided by Zumthor's location by building over the only thermal springs in Switzerland (O'Grady, 2009).

In eastern culture the spiritual connection between bamboo and the mind, connects the mind to the body, if only on a subconscious level. The search for a material that expresses 'lightness' and 'spiritual delight' led to a study on lightweight constructions. The final decision was to use the material bamboo to reflect this connection between the body and the soul. By using bamboo's natural characteristics of flexibility, strength and light weight a connection between materiality and the concept of the mind can be expressed and achieved. Just as important is that the material must be one suited to the environment. Research into the preservation of bamboo will be addressed, as will the possibility of using industrial bamboo materials (composites) to enhance durability and flexibility. The relationship of these composite materials to the soul will also be discussed.

In order to achieve/express the connection between body and soul in an architectural solution an exploration of bamboo in the New Zealand environment will be carried out. This research is conducted through interviews with members of the Bamboo Society to gain in-depth knowledge of bamboo in the New Zealand context. Investigation of the material will then be done through a combination of experimentation and discussion. This discussion will influence the design when making realistic contemporary decisions in relation to the possible outcomes of using bamboo as a building material.

The resultant design will be a combination of ritual bathing, material connections to the body, and a bamboo connection to the soul, which also utilises the natural geothermal resources of the location. 


\section{Bathing and the body}

The aim of this chapter is to describe the bathing ritual and process of public bathhouses, in order to better understand the significant features needed in a design solution. The fundamental connection between the body and the bath, and how each bath type addresses this will be identified. This will be achieved by undertaking research into four types of historical bath, and focusing primarily on how the patrons bathed. The baths selected are considered common and are placed in chronological order, with the exception of the Japanese, which has developed separately from western bath history. Public bathhouse culture began in early Greece, before being adopted and adapted by the Romans. Throughout the ages the popularity of the public bathhouse fluctuated, from Greek to Medieval bathhouse, and even the Japanese bathhouse.

\subsection{Greek Baths}

Bathing, in ancient Greece, was valued not just for its practicality, but also as a means of relaxing the muscles. Most importantly it was also seen as a method for keeping the mind and the body in harmony. The ritual of communal bathing was reserved primarily for athletes post exercise, although there are indications that public bathing became a part of the wider Greek society. Throughout these ancient times, while bath architecture became more elaborate, the primary ritualistic function of bathing remained the same.

In early Greece the first baths were in the open air, and shaded by olive trees. The shallow circular basins called louterions, were approximately one metre in diameter, and used by the athletes pre-exercise to clean, and post-exercise to wash off sweat-soaked sand. The bath process developed from this simple beginning into shallow bathtubs or hip baths, used like foot-baths, where the athletes could wash themselves with a sponge and pour water over the upper body (Smith, 2007, p. 79). The key turning point in Greek bathing came when hot water 
baths were introduced. This allowed the most lavish Greek bathhouses to include separate rooms for bathing, with cold, warm and steam baths. Athletes would remove the sweat and sand from their bodies with hot water, before entering a vapour bath, a type of steam bath created by using hot stones or white hot iron bars. This was followed by a final wash down with a type of soap in the hot bath, and then a plunge into a cold water swimming pool. The heated bath also appealed to the public, leading to the creation of the first Greek bathhouses. These baths were run either by the government, or by private businessmen and were either free or had a low admission cost.

The nil or low pricing encouraged the general population to bathe and baths quickly became popular as a place of relaxation with many bathers repeating the process several times a day. As a consequence of this, they became a place for socialising. (Bonneville, 1998, pp. 20-21). The baths were nondiscriminatory, which also made them a perfect place for socialising among the classes. In Katherine Ashenburg's The Dirt on Clean, she describes a middle-class Greek (Pittheus) and his bathing experience. "Pittheus bathed there in an individual hip bath, one of up to thirty arranged around the perimeter of a circular room... Games such as dice or knucklebones were available, as were wine and probably snacks."(Ashenburg, 2007, p. 23).

The ritual of communal cleansing as the primary method of post-exercise hygiene for Greek athletes, developed into a form of post-exercise relaxation and socialisation not only for the athletes but for the public as well. The popularity of the baths, can be attributed primarily to the connection between the body and water. Although originally designed for hygiene reasons they quickly became a destination for the relaxation of the wider public. The Greek bathhouse had taken the pleasures/rituals of warm bathing, friendly discussion, and introduced games, and forged them into a hub of recreation, with wine and food, all at a reasonable cost or free. The baths had become a place for the public to enjoy. The Roman's adopted this ritual of hot water bathing and the notion of the bathhouse as a place for socialising.

Page | 10 


\subsection{Roman baths}

The Romans noted that the Greek baths had become a place for all to enjoy and adopted the ritual of hot water bathing and the notion of the bathhouse as a place for socialising. They also adapted the alternate hot and cold bathing experience. As the Romans did not exercise like the Greeks the bath for them became primarily a place for pleasure, with the added bonuses of cleanliness and good health. The Roman obsession with public bathing was reflected in the number of baths built in ancient Rome. 170 baths littered the capital, but even though there were many, patrons usually visited only one regularly (Ashenburg, 2007, p. 37).

The Roman ritual of bathing, while being refined from the Greek bathing ritual, was also remarkably similar. The patron began by leaving their clothes in a niche, or open cupboard, where they were looked after by either the staff, or by a personal slave. They then moved through a vapour bath, which involved several different rooms of varying temperatures. Firstly they entered the warm-air room or tepidarium, before choosing to enter either, the dry heat room of the laconicum, or the humid heat room of the sudatorium, before entering the hottest room the caldarium. If at any point the patron needed to cool down they entered an alcove and splashed themselves with cold water from a vat called a labrum (similar to the Greek louterion) (Bonneville, 1998, p. 24). The vapour bath was followed with the patron's immersion into a large communal pool. This pool was contained in a round alcove and was able to hold a dozen people. The patron could then either fully immerse himself in the pool, or sprinkle himself with water from a basin (Ashenburg, 2007, pp. 28-29). The final act of his bathing experience was a plunge into a cold water pool called the frigidarium (Bonneville, 1998, p. 24). This adaption of hot and cold bathing suited the Romans, and most would repeat the bathing process several times a day.

Like the Greeks the Romans also undertook recreational activities during bathing; as a consequence commercial opportunities surrounding the baths were bolstered. Katherine Ashenburg states, "As baths and their facilities grew more elaborate, Romans often spent most of their leisure hours there. With pools, exercise yards, gardens, libraries, meeting rooms, and 
snack bars, the bath became a multi-purpose meeting point. A place to make connections, do business, flirt, talk politics, eat and drink." (Ashenburg, 2007, p. 36). The popularity of the baths had increased, people's obsession with bathing; and their popularity as a place for recreation soon overrode their former purpose as a place for relaxation.

\subsection{Medieval Baths}

Medieval baths can be seen as adaptations of both the Turkish and Roman baths. They were similar to the Roman baths in using both vapour and immersive bathing however they lacked the ordered ritual that the ancient baths once had. The bathing process included combinations of the vapour bath supplying either dry or humid heat usually in a separate domed room (Ashenburg, 2007, p. 79) and communal hot water bathing also undertaken in separate rooms. The bathhouse catered for mixed sexes, unlike its Turkish equivalent.

The bathing ritual at a common medieval bathhouse was a matter of personal preference as to what style of bathing, and what order the patron desired. The central bathing room was large and accommodated semi-private and communal facilities in a single room. On one side a patron could indulge in a communal hot water bath, while on the other side but still in view, they could have a smaller more luxurious bath (Bonneville, 1998, p. 36). The smaller baths were made of wood and banded with iron, and suited two bathers at a time. Ashenburg states that "Although the Turkish bath did not usually include sitting in tubs, the European version - perhaps inspired by memories of Roman baths - did" (Ashenburg, 2007, p. 79). Bathing in baths of different scales allows this private and semi-private relationship by decreasing the proximity between bathers.

The purpose of medieval style bathing was entertainment and festivity (Bonneville, 1998, p. 38). The most luxurious baths were transformed into banquet halls, where music was played by troubadours, and patrons were naked but wore jewellery, makeup and elaborate headpieces; all whilst enjoying food served on floating trays, or boards spread across the bath (Ashenburg, 2007, p. 83). Commercial benefits were obtained from bathhouses which offered extra services directed toward personal 
appearance. As Francoise de Bonneville writes, "Depending on whether the bathhouse keeper was also a barber, additional services might be available, such as cupping, massage, hair removal, hairdressing, beard-trimming and even, in the case of the barber-surgeon, bleeding" (Bonneville, 1998, p. 36).

Medieval baths incorporated the fundamental elements of communal bathing, and the social atmosphere that public baths are renowned for. However during this time there was a dramatic shift from the ethos of the early Greek baths as places for bodily relaxation and friendly discussion, into the bath as a place for recreation, celebration and commercialization. Greek, Roman and Medieval bathing culture all allowed shared bathing, something shared by the Japanese outdoor baths (Smith, 2007, p. 168).

\subsection{Japanese Baths}

The Japanese view bathing as a necessity not only for hygiene reasons but also as a type of spiritual purification, cleansing the mind and the body. There are several types of bath, the hot-water sento, and the geothermally heated onsen, for which there are two subsets, the roten-buro or outdoor bath, and the uti-buro, or indoor bath. While their design may differ in a variety of ways, the common ritual of washing before bathing remains the same for all. In sento bathhouses patrons get dressed (or undressed); wash themselves down removing all suds before entering the bath, as it is considered an insult to dirty the bath water. The more traditional baths have a row of faucets at knee level with buckets to mix the water and soap. One washes oneself with a small towel (tenugui) on a low stool before dropping a couple of buckets of water to rinse the suds off (Talmadge, 2006, p. 58). Other facilities offer a shower, soap and shampoo to patrons to wash themselves with. In all accounts it is a sign of courtesy for other bath users, and the pre-wash ritual is located in a position that the bathers can observe that it has been completed. 
Once clean the patron proceeds to the bathing area with their towel. Modern baths retain a traditional depth of $560 \mathrm{~mm}$, allowing a person to sit up to their chin in the water, with a sufficient volume of water to allow them to remain slightly afloat (Bonneville, 1998, p. 187). Larger baths are also found especially in uti buros, accommodating up to sixty or seventy people at once. Because the washing and shampooing is done outside of the pool, the bath is very clean, and free of contaminants (Grilli, 1985, p. 154).

Once bathing is completed it is common courtesy to share in a post-bath routine of beer and sushi, and engage in a board or card game, most commonly the game 'Go' (Bonneville, 1998, p. 184). Mixed bathing was once common in most bathhouses; however with the introduction of Western culture, the authorities have restricted bathing to same sex bathing facilities. Rotenburos are the only exception to this, where bathers bath in the freedom of nature (Grilli, 1985, p. 156).

The Japanese baths encourage the fundamental purpose of bathing for relaxation, as seen in the early Greek baths. The connection between body and bath in this case is referred to in the sense of 'spiritual purification' that both the body and the mind are cleansed. The Japanese bath ritual differs from the previous (historical western) baths because of the process of washing before bathing. This is seen as a common courtesy, and possibly more hygienic for fellow bathers. The main purpose of these communal baths however remains the same - to socialise, with either friends or neighbours.

\subsection{Summary}

There are common trends throughout the bathhouse rituals of the four studied baths. In the Greek baths alternate temperature bathing was a fundamental element of the bathing process. The Romans had seen the potential in the hot and cold bathing experience and further adapted the Greek's ritual of bathing to accommodate this. In their later bathhouses the Greeks increased the significance of the bathhouse as a place for socialising while the Romans further adapted and commercialised the activity by adding food and leisure as part of the ritual. Medieval bathhouses exaggerated the 'food and leisure' aspects turning the baths into

Page | 14 
places of celebration, and recreation where the focus was on 'entertainment and festivity' (Bonneville, 1998, p. 38).

Both the early Greek baths, and the Japanese baths respected the fundamental notion of cleansing both body and mind. The Japanese however were more refined; for them bathing at the bathhouse was fundamentally for relaxation and cleansing the soul. 


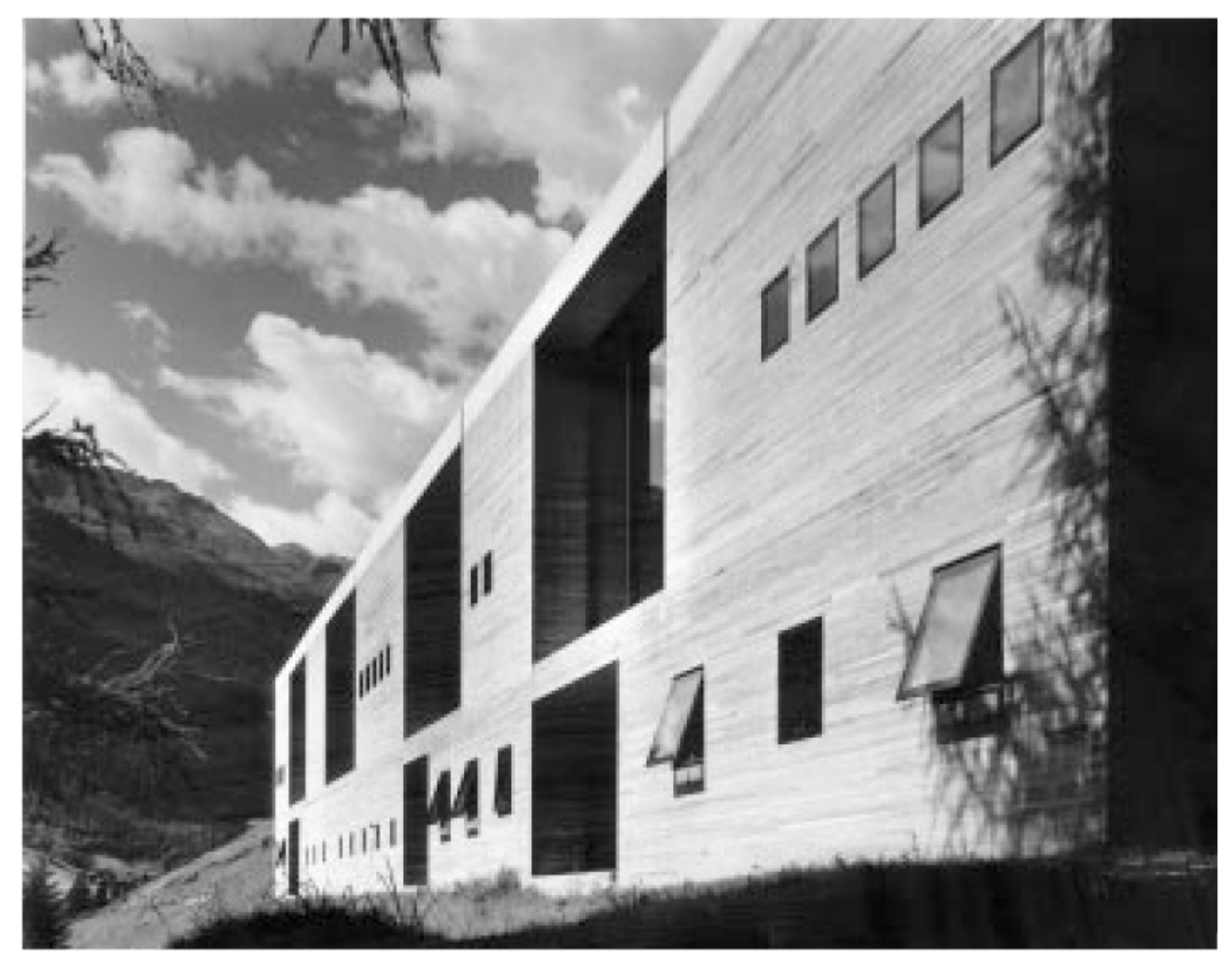

Above Therme Vals exterior highlighting the heavy weight of the building (Spier, 2001, p. 16)

Page | 16 


\section{Bath and stone}

The connection between the body and architecture, or bathing and material, can be used to connect the body and the bath.

This chapter emphasises the importance of materiality and the body, as defined in Peter Zumthor's contemporary bathhouse Therme Vals. Zumthor has carefully addressed ritual in his design through his irregular spatial arrangements. His chosen location and concept, work in harmony to address the connection between the human body and the material stone. The spaces do not follow a prescribed course, or follow any linear planning process. Therefore his spatial arrangement provokes a sense of curiosity in the user.

Zumthor addresses the fundamental premise that bathing is, in its origin, a process for achieving mental relaxation, stating, "In all cultures hot baths are associated not with cleansing but with relaxing, or actually their cleansing is not of a physical but of a mental nature, one usually practiced as a communal ritual (Hauser, 2007, p. 61)." Similarly the Greek and Japanese baths follow the same focus in relation to providing a calming space.
Zumthor achieves this 'calm space' through the use of a variety of bath temperatures, but also through the material selection. By connecting the body to the variety of temperatures, the user becomes aware of the changes on the body. "Some aspects of this tradition can be found in the fire bath as well, it relies on the silent, primary experiences of bathing, cleansing oneself, relaxing in the water; on the body's contact with water at different temperatures and in different kinds of spaces; on touching stone (Hauser, 2007, p. 61)." 


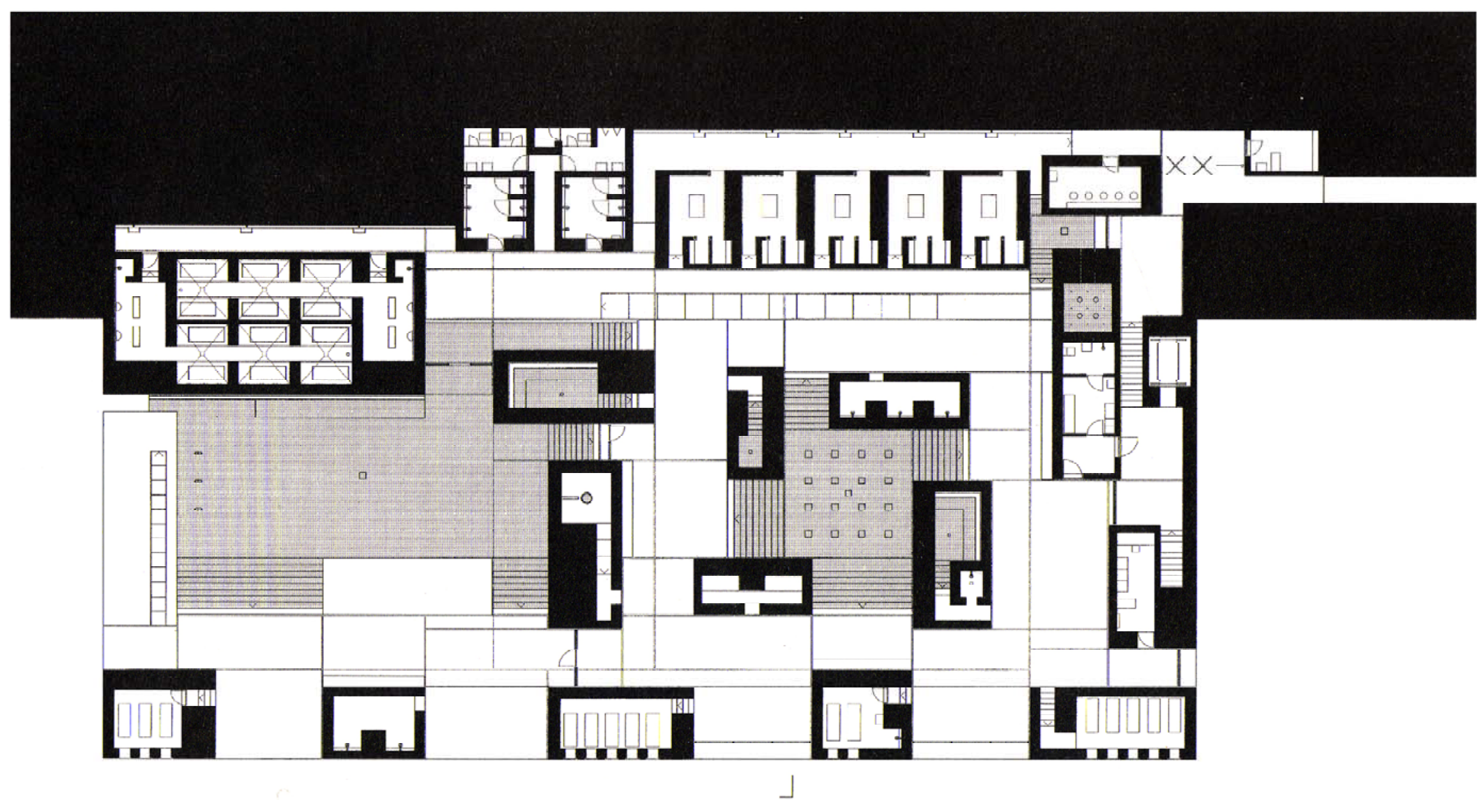

Above Level 1 Therme Vals showing circulation and mass (Zumthor, 1997, p. 25)

Page | 18 
Zumthor connects the primary ritual of bathing, with a fundamental base element as part of the four central elements, fire, wind, water and earth. Zumthor uses stone in his structure to reflect earth, and the subsequent connection between the thermal waters of the bath and the earth which they came. The baths connection to the material is important in recognising the importance of the ritual, "close contact with stone and water; immersing oneself in water for relaxation, as a ritual (Hauser, 2007, p. 89)." Zumthor's conceptual rationale regarding the use of stone and body came from a variety of sources, including the resources available, and the connection with the source of the bath (geothermal water).

The primary motivation for the project's concept, is described by Zumthor "... what seemed to us as a more important role: [was] the establishing of a special relationship with the mountain landscape, its natural power, geological substance and impressive topography (Zumthor, 1996, p. 9)." From this a set of key words was established - mountain, stone and water (Hauser, 2007 , p. 57). His concept established the notion of "boulders in water" and a connection between the local stone and the mountain was formulated (Hauser, 2007, p. 27). The geological substance and mineral connection, as well as the topology, are a sufficient reference for the use of stone. The naturally heavy material connection between stone water and body is therefore justified. 


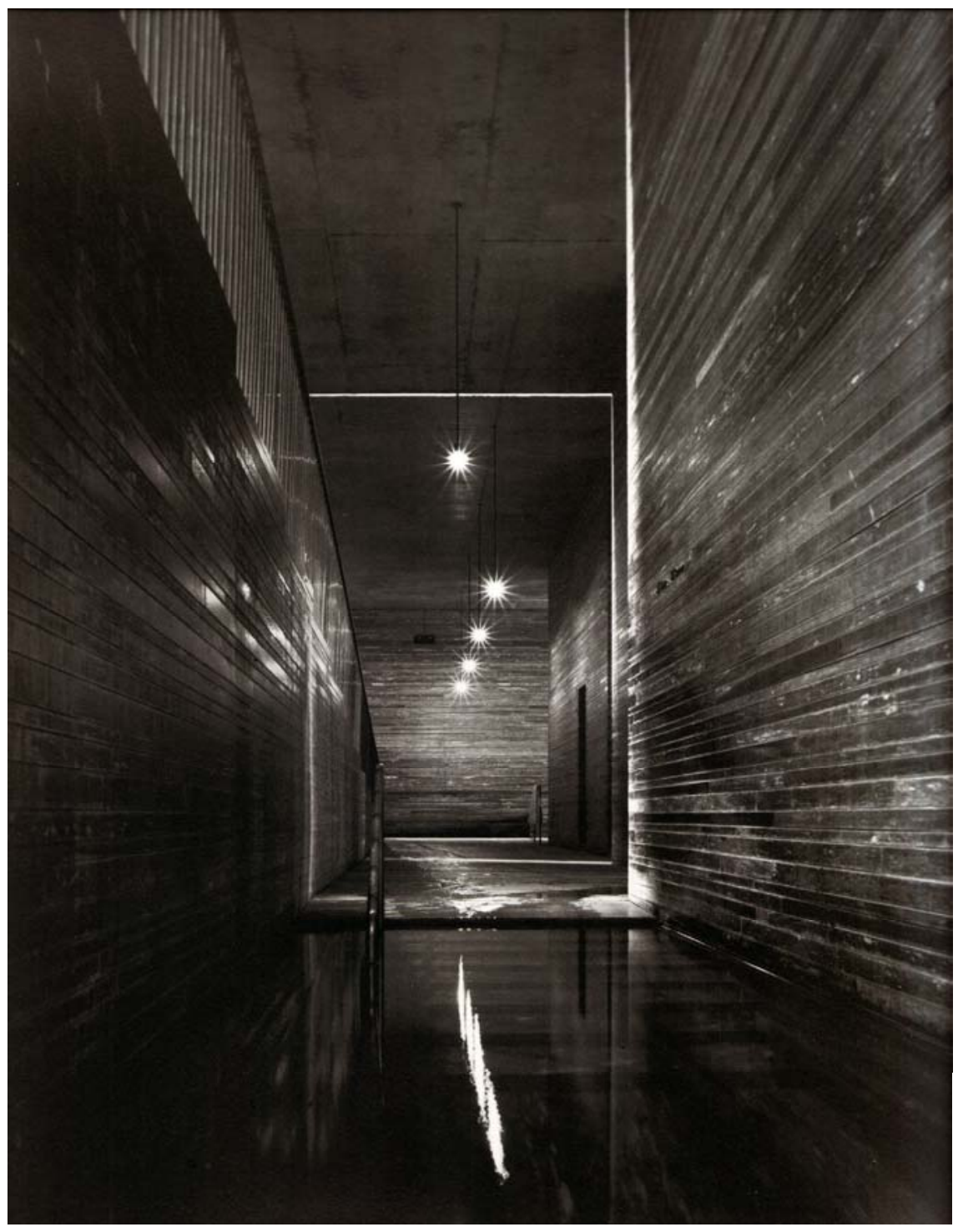

Above Confined enclosed spaces of the interior baths, note geological references in the stone structure (O'Grady, 2009, p. 158)

Page | 20 
The architectural result was a connection between the ground, the geothermal, the mineral, the water and the body. The masses are large and heavy in appearance, and the spaces between large and the cantilevering enormous (Hauser, 2007, p. 47). This is how the architectural connection is experienced in terms of mass and size for the user. Hauser states, "In architecture, stone and water can enter into a natural, and even charmed relationship. Stone loves water, and water loves stone, perhaps even more than any other material (Hauser, 2007, p. 140)." The connection to the mind was a result of the connection the user felt within the space - their individual connection. As Hauser states, "Purification. Peace. Serenity. No noisy attractions, no intrusive stimulation, only the sensation of ones own body undergoing subtle change (Hauser, 2007, p. 89)." Thus Zumthors Therme Vals bathhouse translates and illustrates the connection of material to the body of the user. 


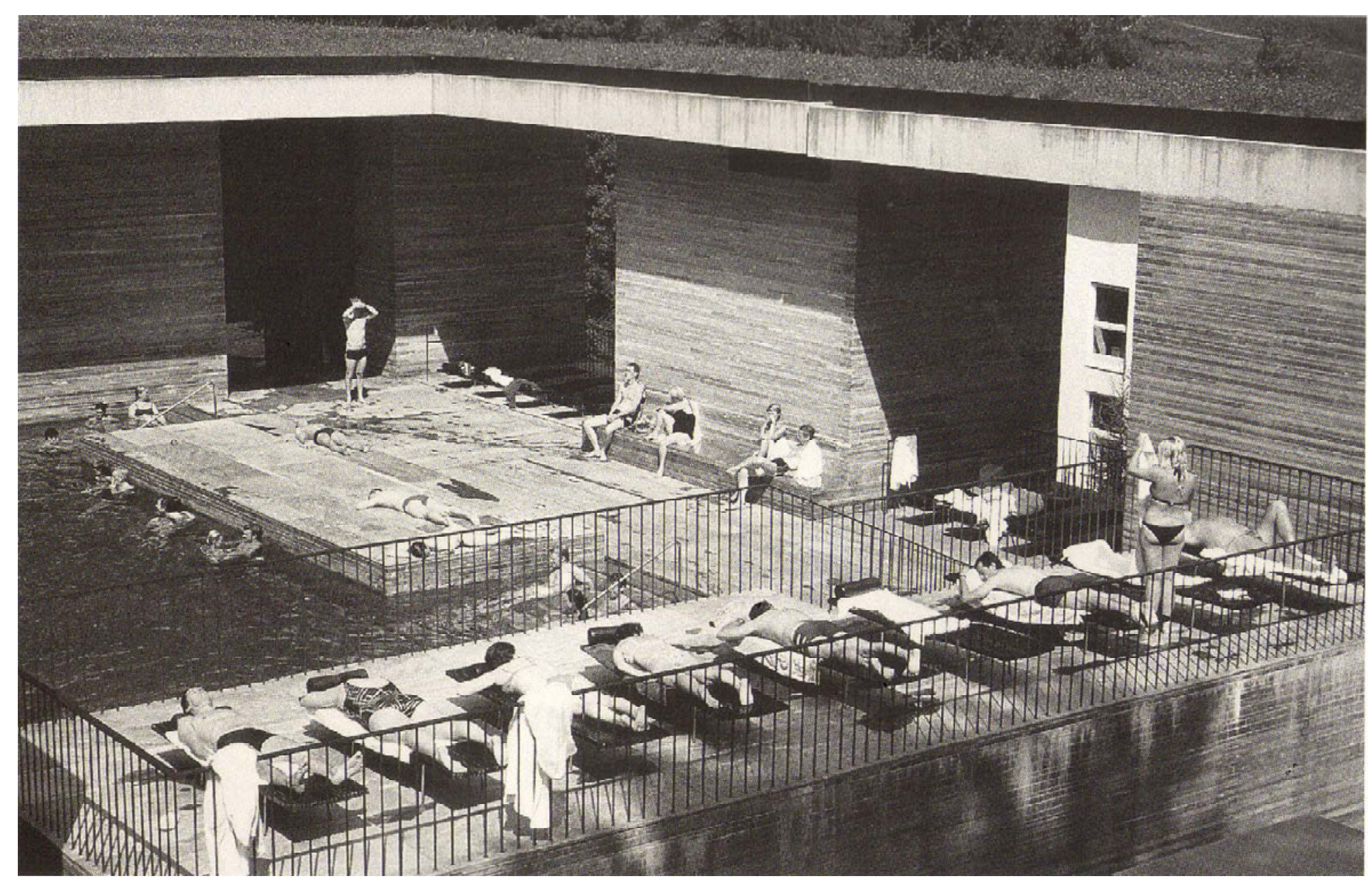

Above Outdoor bathing area showing relationship between architecture and bath (O'Grady, 2009, p. 180)

Page | 22 
At Therme Vals, it is evident the connection between body and water, and stone and water is the seamless reflection of the function or ritual of the thermal baths. The public bath needs to respect the connection between the heavy grounded massing of earth, and the body that inhabits it. The connection between the soul of the user and the baths is respected on an emotional level, and encourages the question of how can materiality reflect this aspect of a thermal bath? 


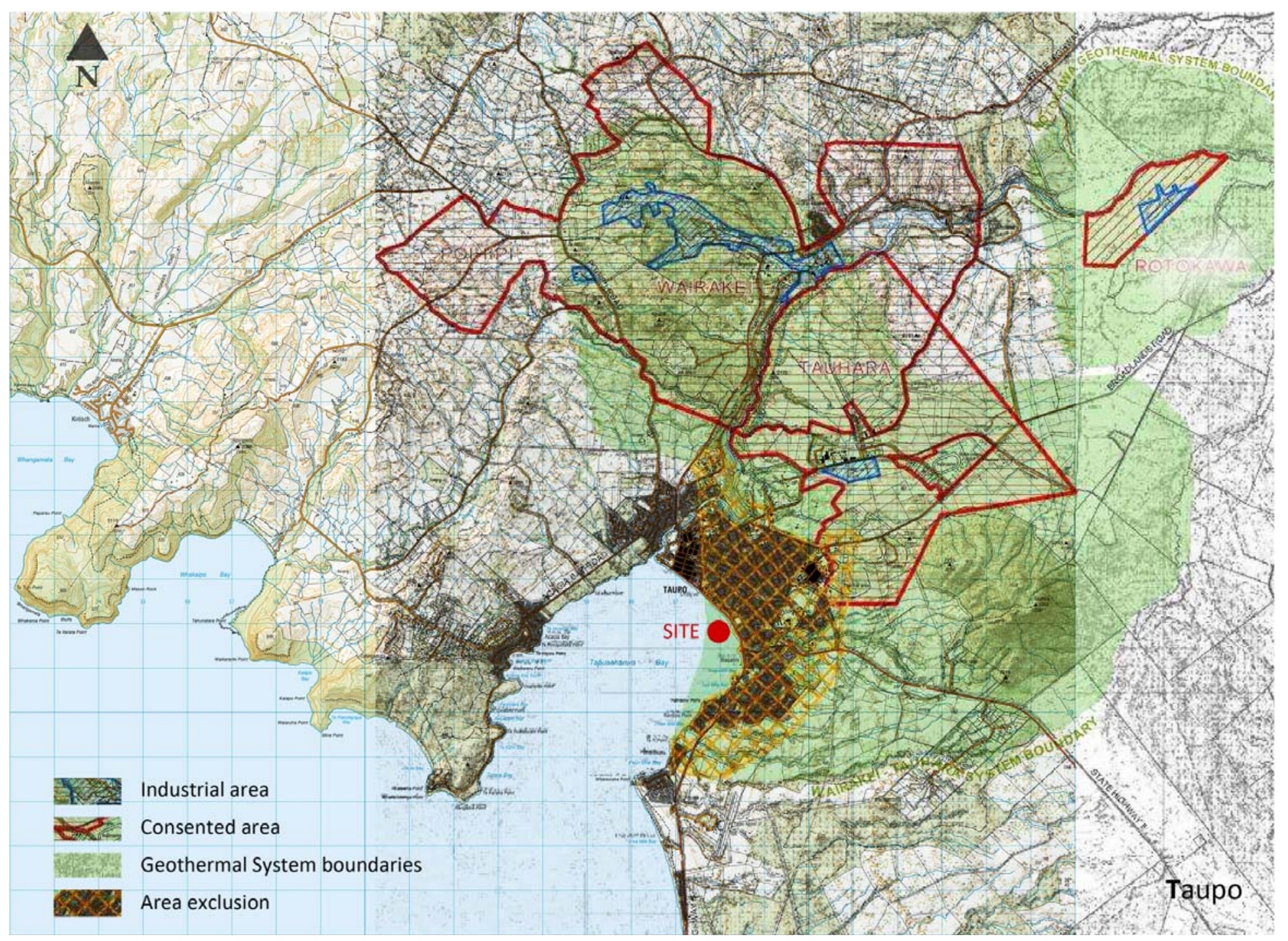

Above Geothermally active areas in the Taupo region (T.D.C, 2010, p. 6) Page | 24 


\section{Town, stone and water}

The selection of the site at Taupo is because of its relationship to the geothermal resources available in the area, reflecting Zumthors location for Therme Vals, the location in relation to the natural geographical landscape, and the relationship to the lake.

Zumthors Therme Vals was "built over the only thermal springs in the Graubunden Canton in Switzerland (O'Grady, 2009)," and therefore the site will reflect this, being the most geothermally active area in New Zealand. The waters of Lake Taupo fill the crater of a large collapsed caldera of an ancient volcano. It is reported that the volcano has produced some of the most violent eruptions ever on the planet (Dench, 2006, p. 7). The geothermal landscape contributes to several exposed pockets of thermal water, particularly in the Wairakei and Tokaanu areas. The geological connection between the lake and the volcano (both cold and hot water bathing) can then be considered a reasonable rationale for selection for a public thermal bath.
The geological and topographical references to the landscape can be viewed from throughout the Taupo district. The lake itself connects the Snow-covered mountains of Ruapehu, Tongariro and Ngauruhoe in the south, the ominous Kaimanawa ranges to the east, and in close proximity the large hill of Tauhara overlooks the township of Taupo. To the West are small isolated bays, where shear bluffs of stone rise from the lake, giving way to gentle countryside before again meeting the Pureora forest (Dench, 2006, p. 5). 


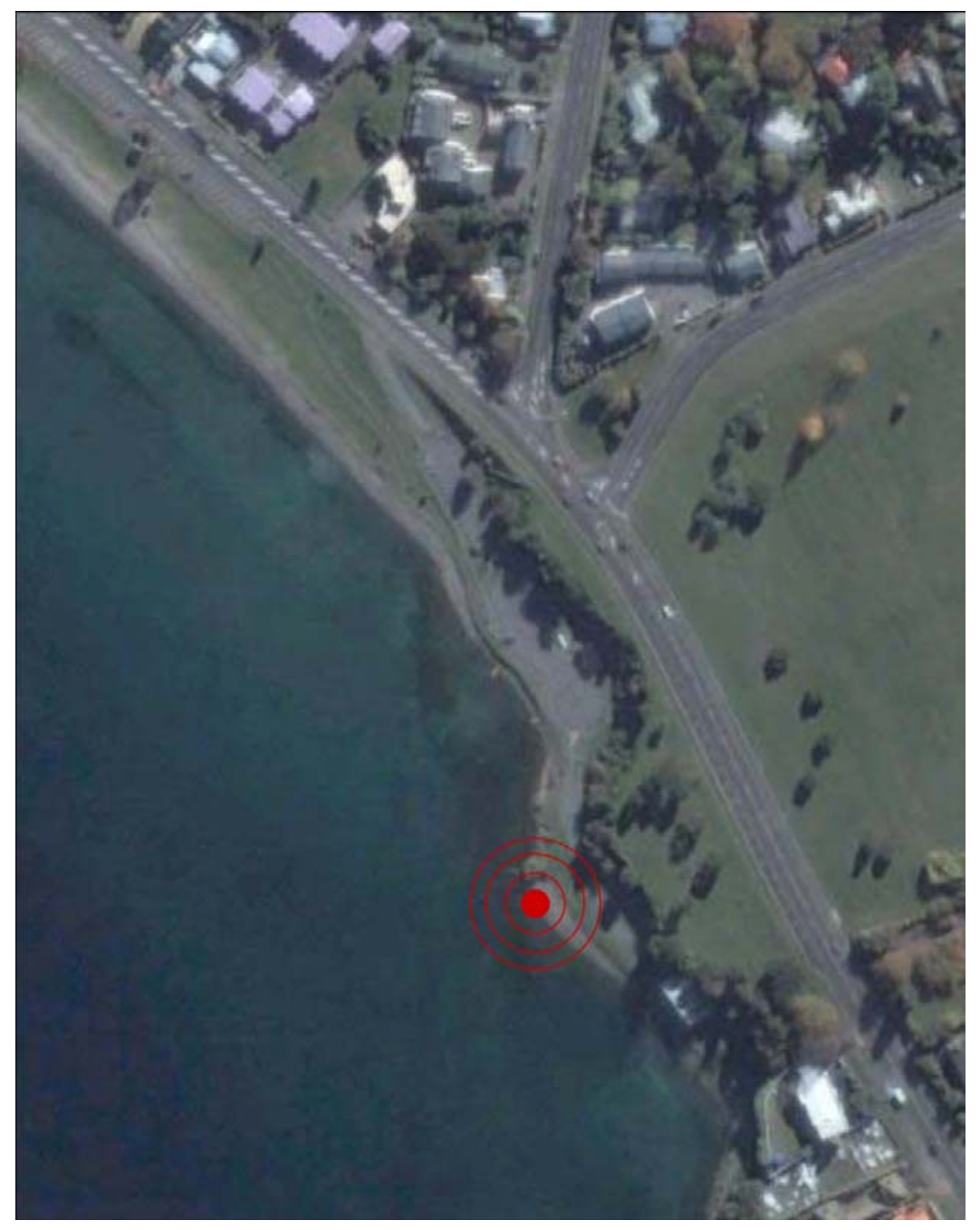

Above Site illustration highlighting car parking, facilities and location of site (Google, 2010)

Page | 26 


\subsection{Site and water}

The chosen site is unique as it bridges at an area where natural thermal waters connect with the lake at two points on site. Located at the south-eastern end of the town beach the site is currently unused. It has some infrastructure already in place including car-parking, toilet and changing facilities. The site is already recognised for its natural shallow hot water pools, however these pools are ankle deep and primarily used only by children (Dench, 2006, p. 34). Underground reservoirs can be utilised to pipe pressurised thermal water to the surface to create the pools. 
The material of the soul

Page | 28 


\section{The material of the soul}

The natural material stone and its connection to the body that is explained previously, connects the body to the material stone. To define the difference between body and soul, a material contrast is needed. The material should therefore compliment the lightweight, and be naturally occurring to reflect the desired composition of the design.

Although a lightweight appearance can be achieved through the use of slender steel members and tension based construction, this lacks an inherently natural appearance. On the other hand, timber has a natural appearance but lacks the slender structural design that is desired in a lightweight design.

The only reasonable material that compliments both the natural and the contrasting is the lightweight grass material bamboo, which can be used in its natural form and in a processed product. The material itself also shares a common relationship with the soul in a spiritual sense. 


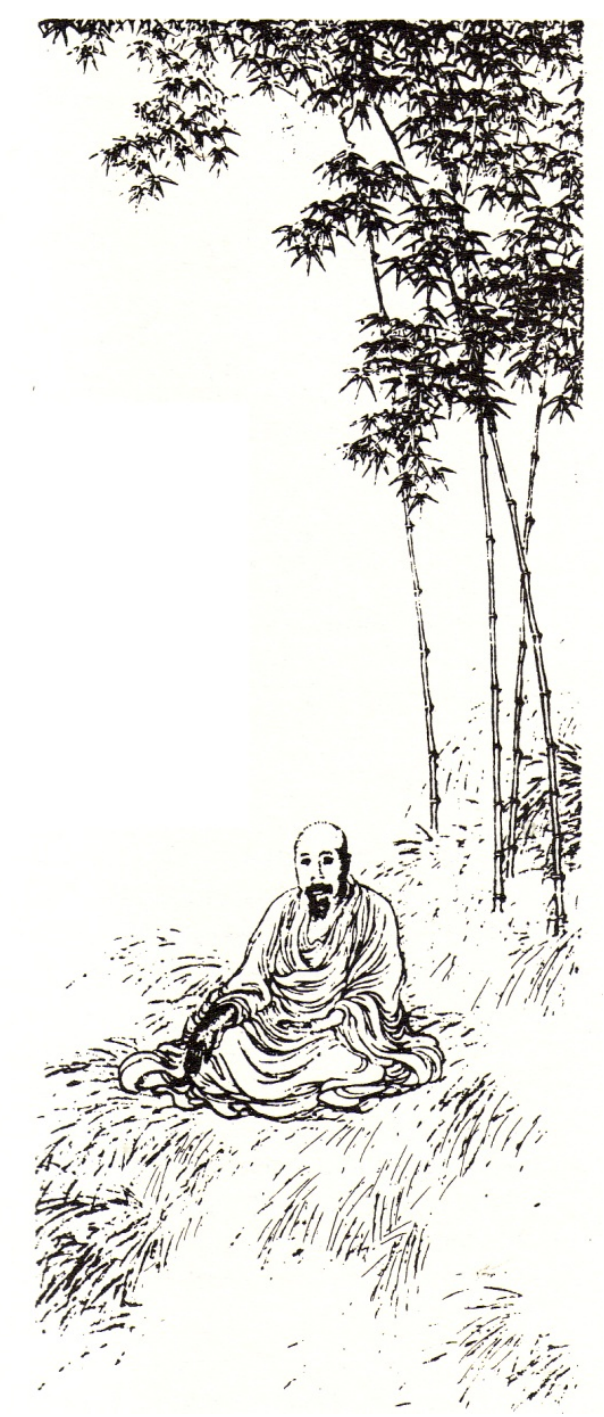

Above The 'mind resort' of training buddhas, scholars and exiles, the bamboo grove (Farrelly, 1984, p. 89)

Page | 30 


\section{The soul of bamboo}

The material chosen is in direct contrast to the heavy nature of the stone structuring used in Therme Vals. Bamboo is both a natural and lightweight material with significant connections to the 'soul' of many Eastern cultures. Laozi, The Buddha and Confucius, the respective founders of Daoism, Buddhism and Confucianism, known commonly as the three friends, (McArthur, 2005, p. 136).

In Asia, particularly in Eastern areas, bamboo is considered righteous, and full of good will; as being strong and able, (referring to) which can translate as strength against the pressures of life. Bamboo's ability to bend and withstand the harshest of storms and return to its original form constitutes the Confucian tenet. Artists in particular have recognised the significance of transforming the natural scenery to reflect the moral character of the scholars' spirit, adapting the Confucian notion that 'the gentleman complies the virtuous' (Dongchu, 1991, p. 11).

Bamboo plants differ from other plants by having a hollow internal core, Daoist's use this notion to symbolise the free flow of
Qi (ch'i) inside people's bodies. "Ch'i is the breath, vitality, or life force at the sightless centre of the ten thousand things; it is the flow of energy that sustains all life" (Farrelly, 1984, p. 78). Because bamboo lacks an internal solid structure, the Daoist's believe that $\mathrm{Ch}^{\prime} \mathrm{i}$ is free to flow inside the hollow core. It is therefore associated with how a person's character complements the free flow of Ch'i within himself.

The hollows within the bamboo plant can also be a reference to emptiness. The healthy respect for nothing is reflected in the feeling that silence has a lot to be said for it. In Buddhism philosophical writings are full of quotes that mention this 'emptiness.' "Since words are given, it's good to know when to stop... The path you name isn't the path you can walk... Those who speak don't know" (Farrelly, 1984, p. 88). With the ideas of the hollow core, bamboo becomes a symbol for 'flexible emptiness' an idea regarded by the masters of Zen Buddhism as the centre of spiritual development (Farrelly, 1984, p. 87).

Bamboo was also an important place of escape, a refuge from the everyday. Similar to the purpose of the early Greek, and Japanese baths, the 'silent will' of Buddhism, requires one to 
escape from the everyday human chaos. The Buddha himself chose a bamboo grove as one of the most frequent homes after his enlightenment. This was subsequently imitated by many other Buddhists and scholars in the following centuries. David Farrelly expands on this saying, "the seven sages of the third century (A.D) China, who retired from the hectic pleasures of court life to a bamboo grove where they could give themselves more fully to philosophy, provided a much-followed model for ages after them" (Farrelly, 1984, p. 88). The later role of the Buddhist monastery, some even named after bamboo, "provided an institutionalised format for withdrawal" (Farrelly, 1984, p. 88).

The Eastern doctrine has particular connections to the spiritual characteristics of bamboo. The material is respected for both its strength and lightweight characteristics, complimenting both the strength of character, and the positive flow of $\mathrm{Ch}^{\prime} \mathrm{i}$. Bamboo plays a role in providing a context from which to achieve a sense of emptiness, as seen in the Buddhist doctrine and Buddha's place of retreat. The material bamboo directly connects the material to the soul, mind, and spirit, through its use in a calming peaceful environment, which is reflected in Buddhism.
The materials ability to bend and sway in the wind, as in Confucian doctrine identifies the bending qualities that need to be explored in a design solution. The bamboo grove is seen as a sanctuary away from the everyday pressures, which is the fundamental rationale for a set of thermal baths, a 'sanctuary' from life.

Page | 32 


\section{The material bamboo}

The purpose of this chapter is to validate the use of bamboo as a material that enlivens the senses. Taken from the idea that the public bath stimulates the body and rewards the user with a sense of euphoria, then how can bamboo stimulate the soul? The goal is to use bamboo as a dynamic building material, one that appears to represent the 'soul' as a lightweight and flexible architecture, a material to contrast the static stone structure of the 'body.' Understanding the unique attributes bamboo has to offer in architecture such as its weight and flexibility will help assist in this. It is also necessary to acknowledge / Justify bamboo's suitability in this environment; its ability to withstand moisture is an important of this. Various industrial products currently on the market are identified as possible architectural solutions.

\subsection{Material characteristics}

Utilising bamboos natural characteristics as a primary form driver, will explore the potential for bamboo in design. Can bamboo appear dynamic in nature to supplement the notion of a bathers 'soul' or mind? Oscar Hidalgo-Lopez describes how in an earthquake bamboo bends and sways, because of its strength, lightness, and flexibility. "The resulting inertia from the earthquake forces, means there is less weight for the walls to support (Hidalgo-Lopez, 2003, p. 357)." The unique feature of bamboo is its inherent flexibility, the ability for culms to bend and sway in the wind and return to an upright position, is unique to the plant. Adapting this quality into an architectural solution is desirable. A.V. Herausgeber provides, in the following quote, some useful unexplored ideas to test in the design:

"The radius of curvature, which changes along the cane according to its circumference, influences its form which can then be put to constructive use. This brings about another aesthetic element in bamboo construction, examples of which can be found here and there in traditional architecture. The architectural potential and scope for manufacturing technology in such bamboo constructions have not been properly analysed and could hold unexpected potential (Velez, 2000, p.221)." 
The idea of utilising the full bamboo culm's inherent flexibility in a multi-directional curve will appear both dynamic and lightweight in comparison to the strong rigid mass of the baths 'body.' Once the bamboo is bent it can be used in a range of different forms, and is not restricted to bending in a single direction. As Herausgeber states, "Thin canes can be bent when dry, whereas thicker canes are bent wet and then dried. Some structures utilising dry bent canes have an asymmetric curvature, which increases as the canes' thickness decreases, because the flexibility of whole bamboo canes decreases with mounting thickness." Once the curvature of the bamboos exceeds a certain point, split bamboo is recommended because it requires less effort in bending (Velez, 2000, p.215).

Bamboo can showcase an exceptional flexibility; a necessary dynamic for expressing the lightweight nature of the design. The ability for the material to bend and flex, even respond to the environment through movement, can be extended to a design solution.

\subsection{Durability}

The durability of the structure is dependent on the treatment solution. There are three basic types of preservation available: The least desirable is leaving bamboo untreated, natural preservation can provide short-term protection, but for the long term, chemical treatment is required in most situations.

Bamboo is a natural material and behaves in similar ways to timber when exposed to the elements. In areas where bamboo is a dominant resource, mainly in tropical environments around the equator, bamboo has a life expectancy of less than five years. Lopez describes that, "Bamboo under ordinary conditions has an average life of 1-2 years when exposed to the atmosphere and in contact with the ground; while a service life of 3-5 years can be expected from bamboos used under cover, and out of contact with the ground. When used indoors from 6-8 years." (Velez, 2000, p.142). The reason why bamboo has such a short life expectancy is to do with the presence of a starch interior, which makes bamboo susceptible to micro-organisms and rot without preservation (Preservation of Bamboo, 2006, p. 12). Cracks and 
splits increase the degradation of untreated bamboo by allowing entry points for micro-organisms. Reducing splitting due to nailing without pre-drilling and sudden drying is a priority (Preservation of Bamboo, 2006, p. 19). Most importantly increasing the life expectancy through preservation is considered more economical in the long run (Preservation of Bamboo, 2006, p. 12).

Natural treatment solutions have limited success in preserving the bamboo, depending on its use. Because of its waxy exterior bamboo preservation differs from timber, as traditional timber treatment methods are ineffective in penetrating the waxy surface. There are traditional immersing techniques, such as submerging bamboo in stagnant or running water, mud, or beach sand for a period of several weeks, removing the starch and sugar content from the culm (Hidalgo-Lopez, 2003, p. 146). Alternatively treating bamboo by smoking or leeching has a similar effect. These techniques are simple and cost effective but do not provide adequate long-term protection in exterior environments. The solution to this is to use chemical preservatives (Preservation of Bamboo, 2006, p. 25).
Chemical treatments provide assured protection against degradation. Although all timber treatments work on bamboo, only some are recommended for round bamboo. Treatment solutions can be broken down into two types, oil, and watersoluble salts. Oil types are used where bamboo or wood is in direct contact with soil and water, but tend to stain the bamboo; therefore it is undesirable in areas where the appearance is important. Water soluble salts preserve bamboo that is not in contact with soil or water, the benefit of this type is that the bamboo can then be painted over and varnished (Hidalgo-Lopez, 2003, p. 148).

A salt based solution will provide the bamboo with an extended life, without affecting the appearance, provided it is not in contact with the ground. A better possible solution is to introduce industrial bamboo materials, which have the benefit of preservation, while being reformed into a more versatile material. 

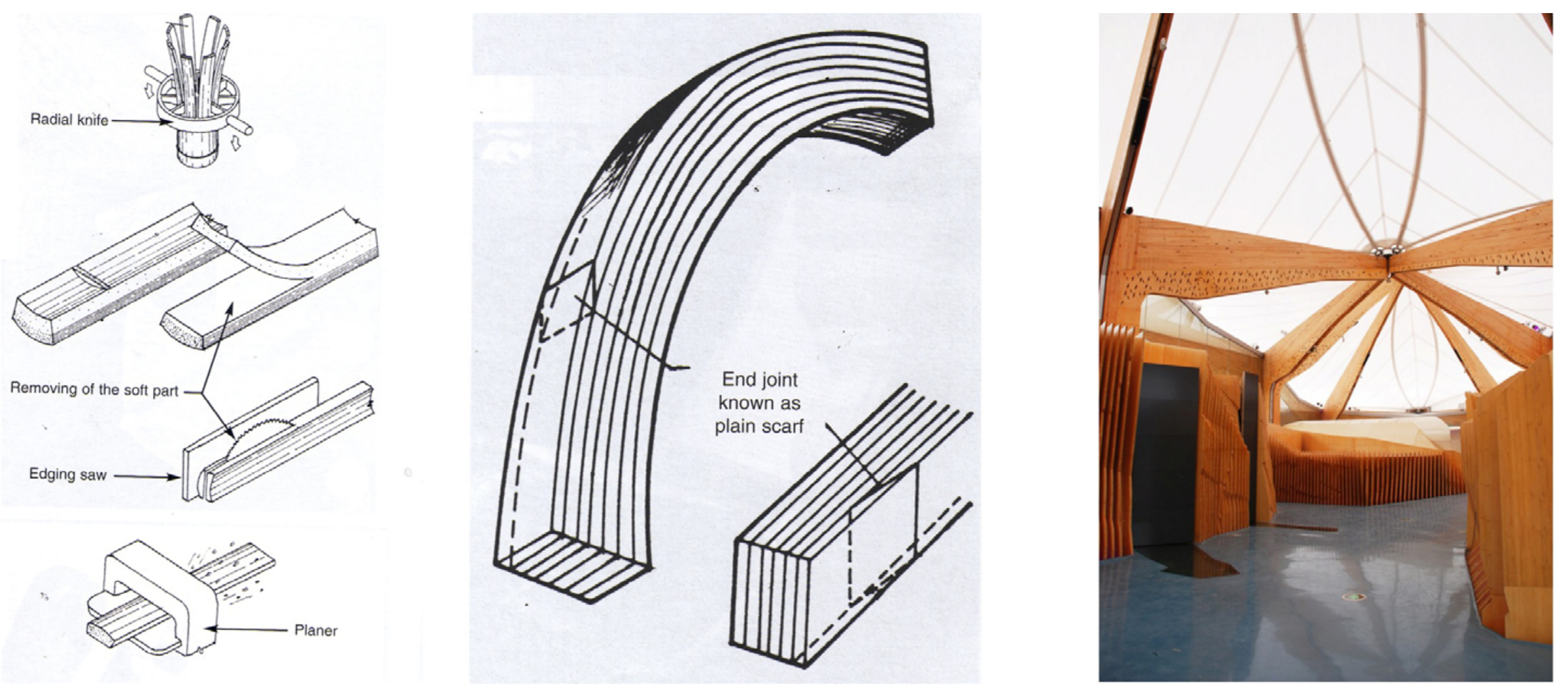

Left Manufacturing bamboo laminates (Hidalgo-Lopez, 2003, p. 190)

Centre Curved laminate beam (Hidalgo-Lopez, 2003, p. 191)

Right Norway Pavilion at World Expo 2010 Shanghai, has a secondary and interior structure of laminated bamboo (Sebastian, 2010)

Page | 36 


\subsection{Industrial bamboo materials}

Industrial bamboo materials, also known as bamboo composites have a wide potential in architecture. The various products have transformed bamboo's properties, strength, and flexibility into solutions that retain these benefits and reduce the shortcomings such as size, shape and multidirectional strength (Qisheng, 2003).

The focus of this section is to explore the most readily available, weather resistant bamboo composites that can be used additionally in the design. Industrial timber solutions of woodbased products and bamboo based products are similar in structure and composition. The strength and rigidity of bamboo products such as plybamboo are higher than ordinary plywood (Qisheng, 2003). As mentioned before bamboo can substitute for wood in many of its uses, and industrial bamboo can further increase its uses in fields such as flooring, wall panels and plywood in construction and architecture (Hunter, 2003). By applying bamboo into a panel form, technical problems relating to bamboo's geometry in construction can be avoided (Pablo van der Lugt, 2007).
The purpose of the following section is to identify potential industrial materials that can utilise the benefits of bamboo's flexibility into a westernised form. Investigations into two types that follow this flexibility and processed form are bamboo lumber (skeleton), and bamboo mat board (skin).

\subsubsection{Bamboo lumber (LBL)}

The most dynamic and adaptable structural application of bamboo composite technology is the laminate structural member. This material combines the strength and flexibility of bamboo into a rigid structural system. Glue laminated (glulam) bamboo is created the same as typical timber laminates, that is several layers of bamboo or wood are glued together with all layers running parallel to the next (orientated). Using this method complex curve's can be achieved by gluing bamboo laminates together inside a formwork. The depth of a laminated beam is easily varied in response to the span required. The width of the beam is achieved by stacking several laminates together, or by using square bamboo grown in formwork to create laminates up to 

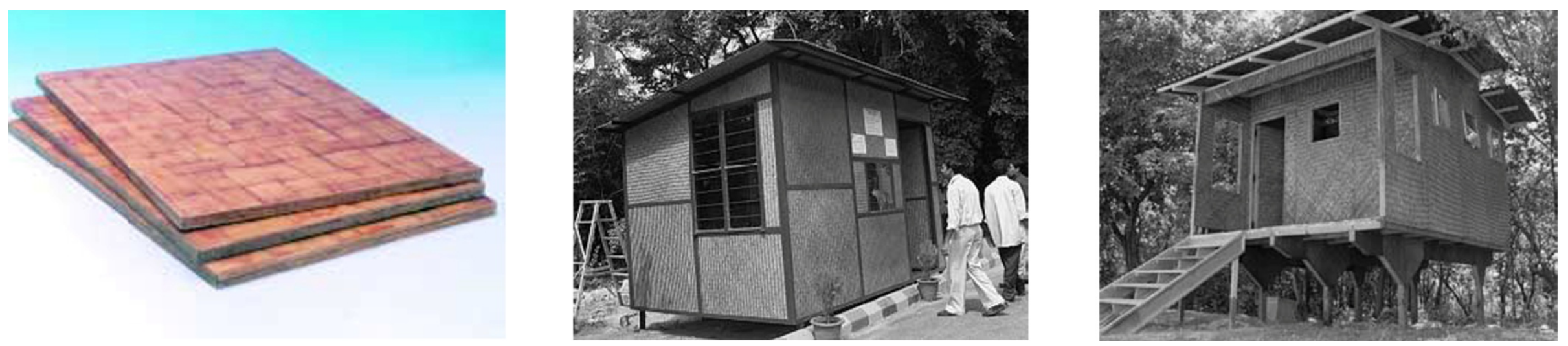

Left Bamboo mat board (Qisheng, 2003, p. 94)

Centre Bamboo matt board cladding on house (Ed. Xiao, 2008, p. 59)

Right Bamboo matt board cladding on house (Ed. Xiao, 2008, p. 59)

Page | 38 
100mm wide (Hidalgo-Lopez, 2003, p. 309). The larger species of bamboo are preferred in industrial applications, Moso bamboo (Phyllostachys pubescens), and Ma bamboo (Dendrocalamus latiflorus) of specific sizes were found to be most suitable (Ganapathy, 1996). The resulting product has high mechanical properties and excellent weather resistance due to the resin adhesives (Wang, 2003). The appearance of flexibility and, dynamic architecture can be achieved with the processing of bamboo into laminated lumber; this allows the forming of complex curved solutions using formwork and adhesives.

\subsubsection{Bamboo mat board (BMB)}

The strongest and most widely used bamboo panel product is mat board. Bamboo mat board has been around for the last three decades but only recently have productivity changes made it widely accessible. It is the first bamboo-based panel to be produced commercially (Ganapathy, 1996).

Mat board is made from strips of bamboo woven together to form a mat. This is then impregnated with a resin compound and bonded together in a hot press. Multiple mats are fused together to form sheets of between three to nine layers depending on the thickness required (Pande, 2008). Valued for its high modulus of rigidity (MOR) and flexibility, it can replace thin plybamboo and plywood in any application (Hidalgo-Lopez, 2003, p. 184).

$B M B$ is stronger, more durable, and more directionally stable than wood based plywood. Panels with this non-orientated structure improve the structural qualities of bamboo, by increasing strength in both directions as well as minimize instability in wet expansion and dry shrinkage (Qisheng, 2003). $\mathrm{BMB}$ panels are durable and resistant to boiling water, extreme climatic conditions, wind, rain, and micro-organisms. They also resist scratching and staining better than the industry standard phenolic-bonded plywood (IPIRTI, 2001, p. 41).

The flexibility of $B M B$ is the main advantage of this product. "... (BMB) is more flexible than equivalent plywood. This property of BMB can be advantageously used in many engineering applications (Ed. Xiao, 2008, p. 60)." The product is uncommon in architecture but has potential for use as stretch skin panels, wall bracing and web beams (IPIRTI, 2001, p. 42). Its current use 
includes cantilever and stressed skin panelling for all types of light aircraft in the Asia region (Hidalgo-Lopez, 2003, p. 432).

$B M B$ then is a durable, flexible material with lightweight characteristics that can be applied to construction in replacement of plywood alternatives.

\subsection{Summary}

The material bamboo is well suited to contrast the heavy mass of the body and stone. It is natural, flexible and lightweight and is therefore perfectly suited to a dynamic architecture that reflects these characteristics. The ability for the material to bend and flex, even respond to the environment through movement, can be extended to a design solution.

It is strongly recommended that bamboo be preserved to increase the life expectancy of the material. There is a range of natural and chemical treatment options for bamboo, most have been adopted from timber treatment however and only a few are recommended for treating round bamboo. The range of natural treatments permits short-term solutions to preservation. For long- term protection chemical alternatives are recommended. Salt based solutions are recommended as they don't stain the culm, provided the bamboo is not in contact with the ground.

Industrial bamboo materials have the benefit of preservation, while being reformed into a more versatile material. Bamboos inherent difficulties in constructability from the cylindrical form are resolved in the processing of the material. The aesthetic qualities of industrial bamboo differ from the existing timber stock, and can provide architecture with a variety of different appearances using the same material. The structural skeleton can be achieved using bamboo laminated lumber to give strength, the appearance of flexibility through curvature. The structural skin can be achieved with BMB, as its durability, flexibility and lightweight properties can be applied to a design that complements the lightweight, dynamic characteristics of the architecture.

Application of bamboo into a design solution for the public bath requires research and application in the New Zealand environment. 


\subsection{Design}

\subsection{Bamboo design process}

In this chapter an exploration of the use of bamboo as a visual representation of the soul is discussed with relation to the potential for a design solution in this area. A combination of experimentation and discussion with experts in bamboo influenced the exploration of the design's potential.

The early design process was a combination of design and full-scale experimentation. Identification of the dominant timber species influenced the choice of material for experimentation. The suitability of bamboo, and the design outcomes are discussed in a series of interviews with New Zealand leaders in the field of bamboo. An assessment of the experimentation is outlined in a summary of the suitability of typical construction connections, the flexibility of bamboo, and how the material behaved in the outdoor environment. After several months, a review of the experiment identified issues that could influence the design.
The outcome of this work is a greater knowledge of bamboo in New Zealand and a better understanding of how the material can represent the light, flexible nature of the soul. 


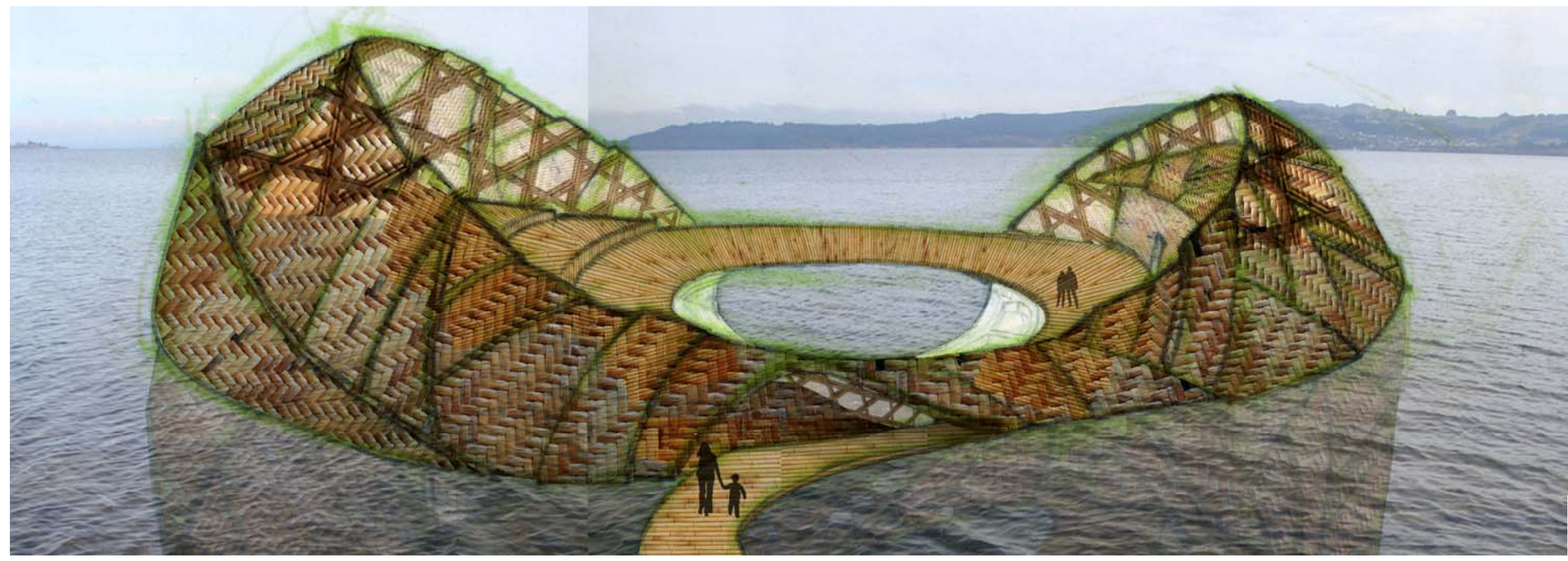

Above Primary concept illustrating bamboo lattice, BMB, and bamboo flooring.

Page | 42 


\subsubsection{Bamboo Concept}

By adapting research from the bamboo architecture section of the literature review, an initial concept involving a sheltered cove was established. The structure is composed of a full culm curved lattice, adapted from Velez' discussion on the unutilised potential of bamboo in its curved form. This is balanced with a contemporary industrial bamboo form of bamboo mat board (BMB) that is valued for its dimensional stability and inherent flexibility. The structure was to be based on a floating pontoon, which would allow the lightweight benefits of bamboo to be utilised, and exploited, requiring less floating substructure and therefore less industrial material.

\subsubsection{Concept discussion}

The structure reflected several important outcomes of the discussions. The appearance of the BMB sheets, woven into the circular form had an unintended cultural connection to the indigenous societies of New Zealand. Removing the inherent cultural connection became a priority of the following designs. The bamboo lattice was adapted and refined for full-scale experimentation. 


\subsubsection{New Zealand timber bamboo}

Two key figures in the Bamboo Society of New Zealand, Mark Mortimer and David Hyams assisted in discussions throughout this chapter. Mortimer described the dominant timber bamboo species as:

“Bamboosa Oldhamii (clumping) - Used in shelter belt situations, which grows erect, and is common around northland. Branching occurs all the way to the base of the culm, which can be problematic and should be designed for as extra work is required in pruning the branches. The resulting culm has branch defects at the join. It is thick walled very strong, and most importantly available. It is not good for splitting.

Dendrocalamus latiflorus (clumping) - larger culmed variety than Bamboosa Oldhamii. Not as common as Oldhamii, and is not really used in shelter belt situations. Grows well in Northland and warmer areas of New Zealand where it grows up to $120 \mathrm{~mm}$ diameter.

Phyllostachys nigra henonis (running) - sub variety of Phyllostachys nigra, though much larger in diameter. It is the most available large scale running bamboo in New Zealand. It is a thin Page $\mathbf{4 4}$ walled variety, but very strong because of its density. Splits very easily and cleanly, therefore it's ideally used as lathes. $80-100 \mathrm{~mm}$ in diameter.

Phyllostachys pubesens (Moso) / Phyllostachys edulis (running) - The best bamboo in terms of quality of material" (M. Mortimer, 2010). David Hyams added, "The second largest introduction of bamboo occurred in the Taranaki region, with the introduction of Moso from Japan; the successors of the germinated Moso still remain in the area." Moso can easily grow up to $150 \mathrm{~mm}$ in diameter in the right conditions (D. Hyams, 2010).

Bamboo is an introduced species in New Zealand, therefore the groves are considered juvenile in age. Hyams stated that, "Bamboo is in its early growth in New Zealand when compared to other Asiatic countries." New Zealand does not have the availability of large stocks of bamboo like other countries. The feasibility of industrial processing is limited without industry and silviculture development. (D. Hyams, 2010).

The dominant timber species that can be applied to an architectural or construction solution in New Zealand are Oldhamii 
and Moso. Moso in particular is preferred as it grows without leaves on the lower section of the culm requiring less processing than Oldhamii. The quantities available for large-scale production are currently unavailable, however with proper silviculture management; the potential for industry growth is possible. 

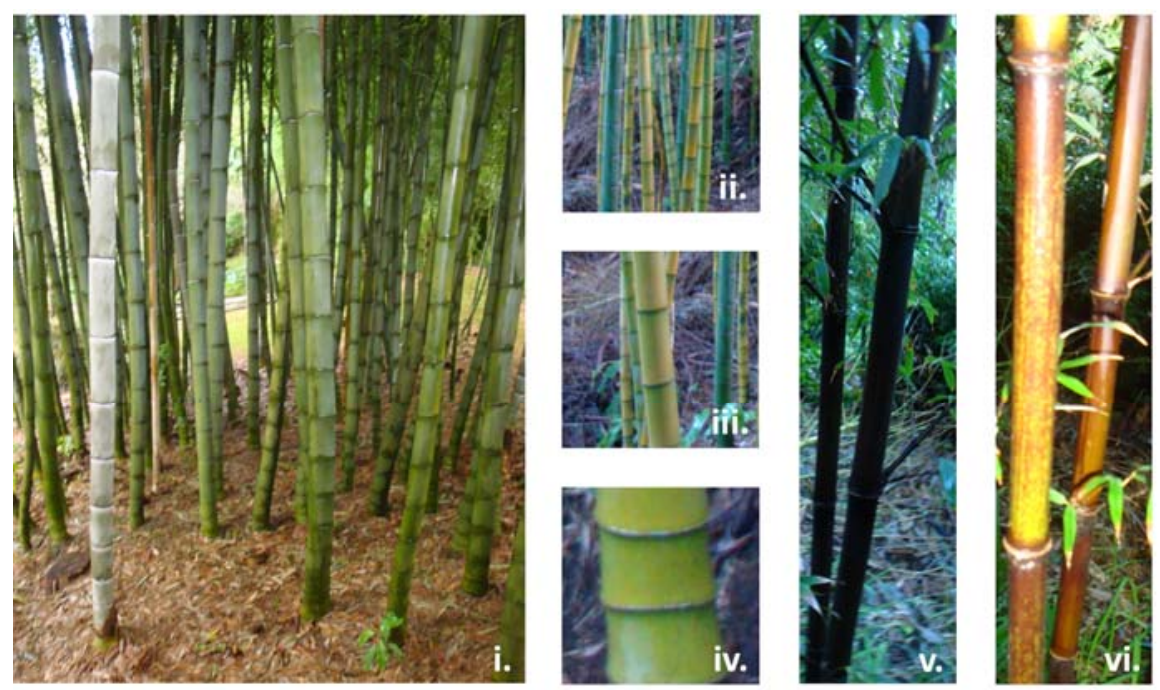

i-iv Moso Bamboo in a maintained plantation Taranaki.

v-vi Phyllostachys Nigra (Black bamboo) Small culm with different colouring.

vii-ix Bambusa Oldhamii The bamboo harvested for case study.

x-xi Harvesting (done with handsaw) and transportation.
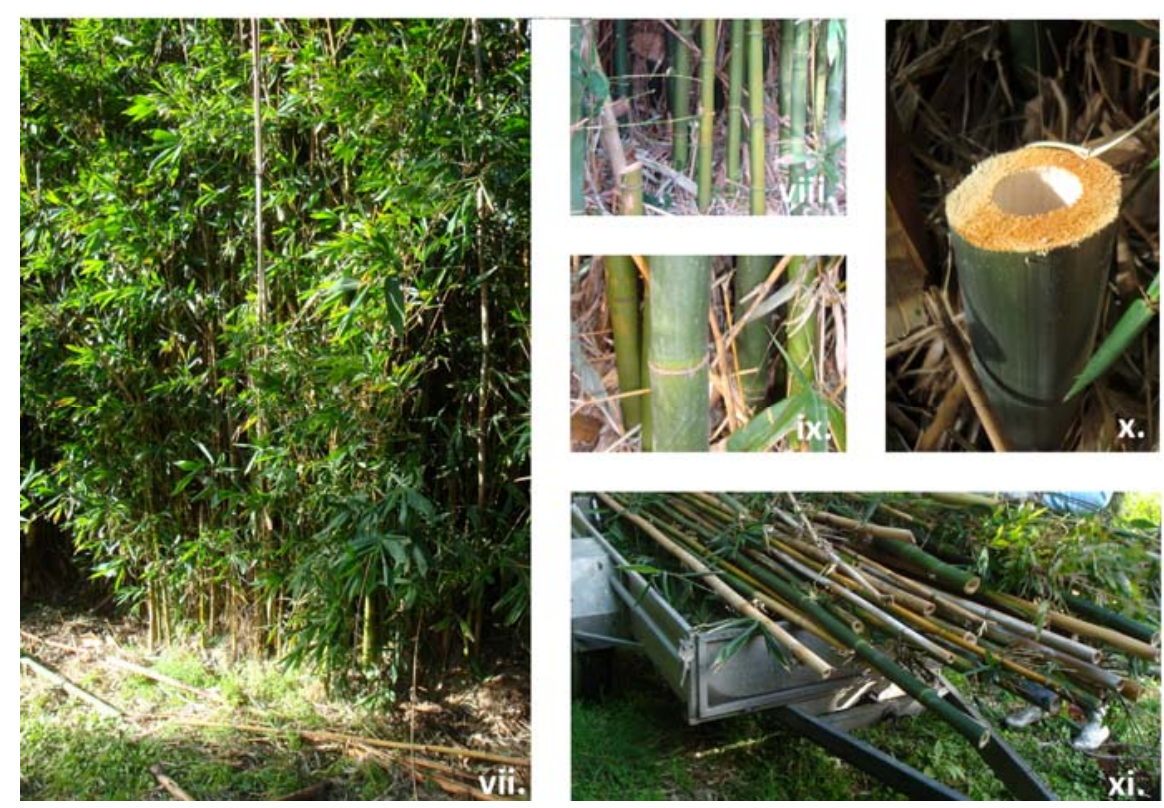

Page | 46

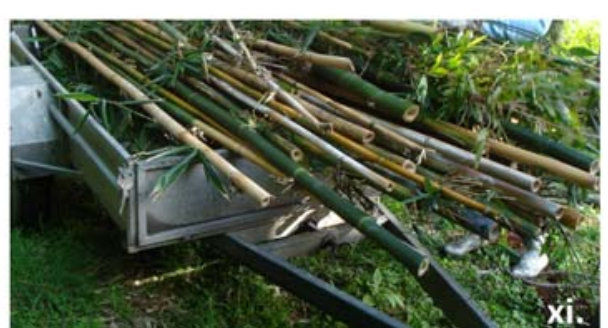




\subsubsection{Harvesting and processing}

The experiments were to be carried out in Taranaki, because of the available space and tools required. The bamboo chosen for the experiments was Oldhamii for its availability and culm diameter of between $\varnothing 50-100 \mathrm{~mm}$, when compared to the available Moso $\varnothing 100+\mathrm{mm}$. The smaller culm size should inherently require less effort in bending and forming.

The time taken to harvest and process the bamboo should not be underestimated. Moso requires less effort due to its branches beginning up to $6 \mathrm{~m}$ higher while Oldhamii requires extensive labour in removing the branches from the culm. Once removed the branches have little use other than fodder for the grove. The other drawback of the Oldhamii species is its appearance once processed, with cut segments alternating up the culm at each node. 

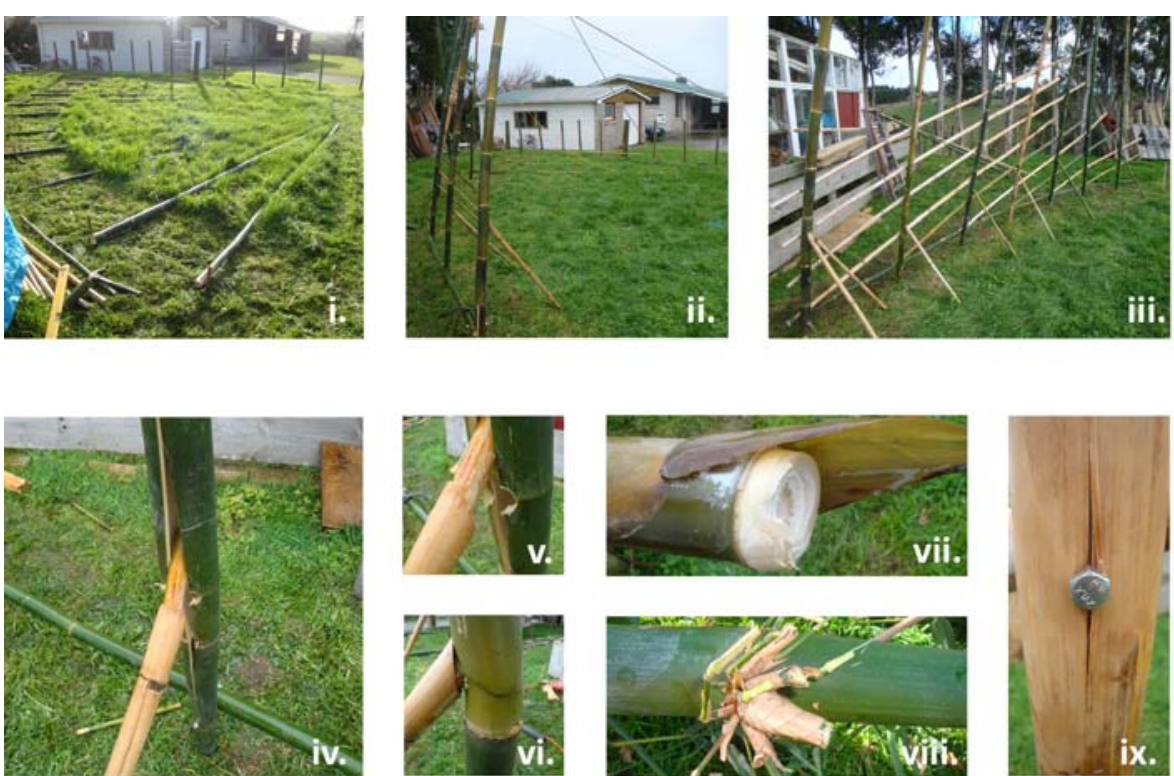

Angled doweling supports, and ropes ensure a suitable bend in wall
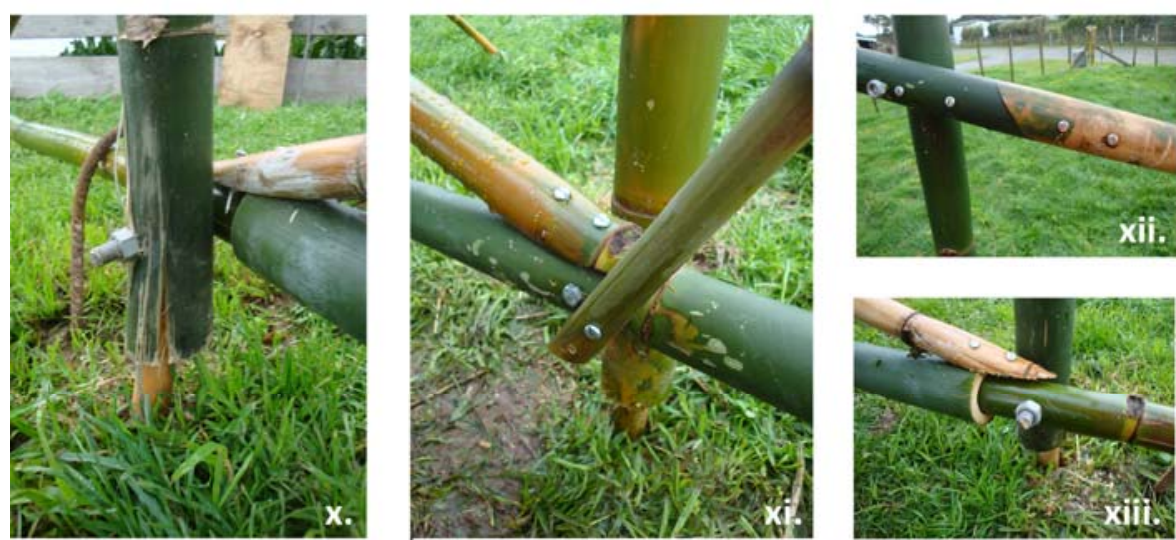

ix Split resulting from dry cum not predrilled correctly

$x \quad$ Splitting at base

xi Compound joint showing three

scales of bamboo and fixings

xii Flush joint from use of digital

Callipers

xiii Combination slip and bevel joiners in

areas under large bending stress

xiv Bevelled finish on culm ends

xv Pan head screw on outer layer

xvi Drainage hole at top node
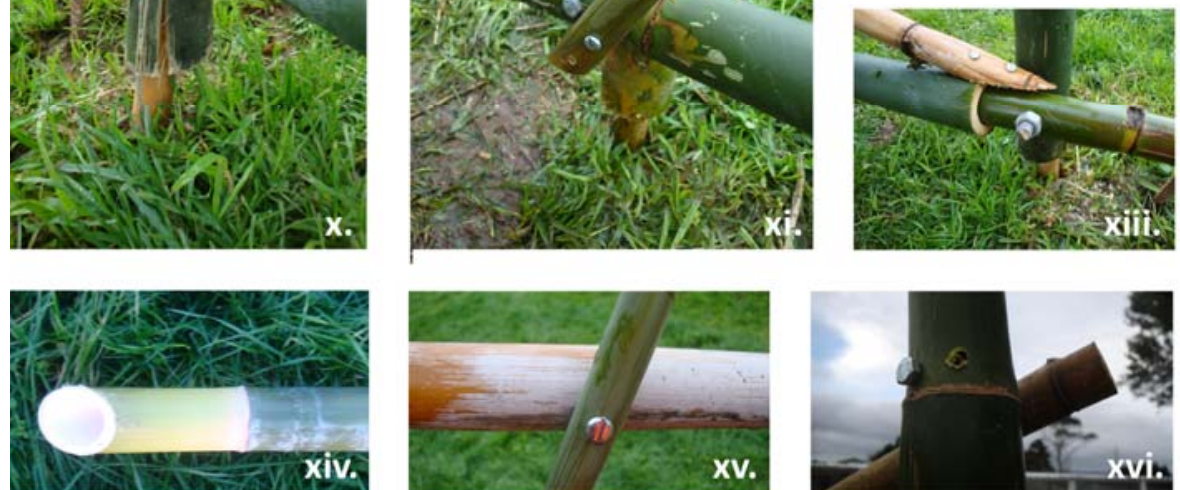

Page | 48

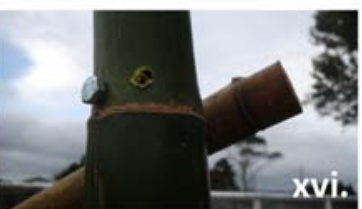




\subsubsection{Experimentation}

The aim of this test was to identify how successful standard joining techniques are when used on bamboo, and how bamboo behaved after exposure to the elements for a three month period.

A variety of bamboo scales were used to demonstrate the potential for variable scaled bamboo support. Both cut and dried bamboo were used to test the bending capability of the two materials. This is important in testing the flexibility of the material, and how it can better express the contrasting curvature of the structure. Also how different scales can be used to achieve a complimenting material composition through the use of a single material in a variety of ways.

Connections between green culms reduced the chances of unexpected cracking, because of the softer bamboo fibres. M6 $100 \mathrm{~mm}$ zinc bolts were used for the majority of the structure, with threaded rods and bolts substituting for fixings above $100 \mathrm{~mm}$. The time involved when using pre-drilling and bolted connections, was significantly longer than using simple selftapping timber fixings, and should not be underestimated.
Connection design should therefore be prioritised when dealing with bamboo construction with reduced culm penetration. 

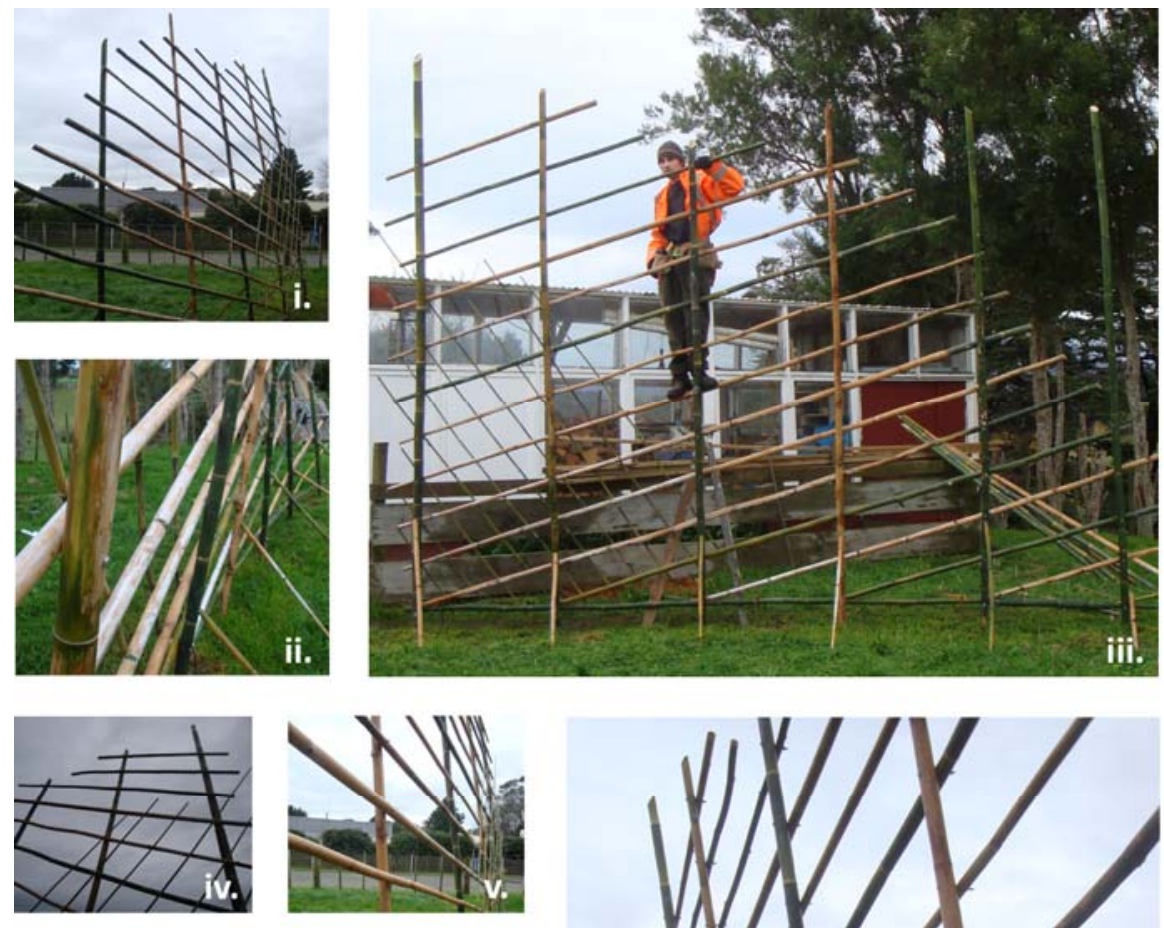

i-ii Finished frame

iii Fixing strength highlighted

iv-v Layered cross members of varied size

vi Doweling support

vii Small curve achieved

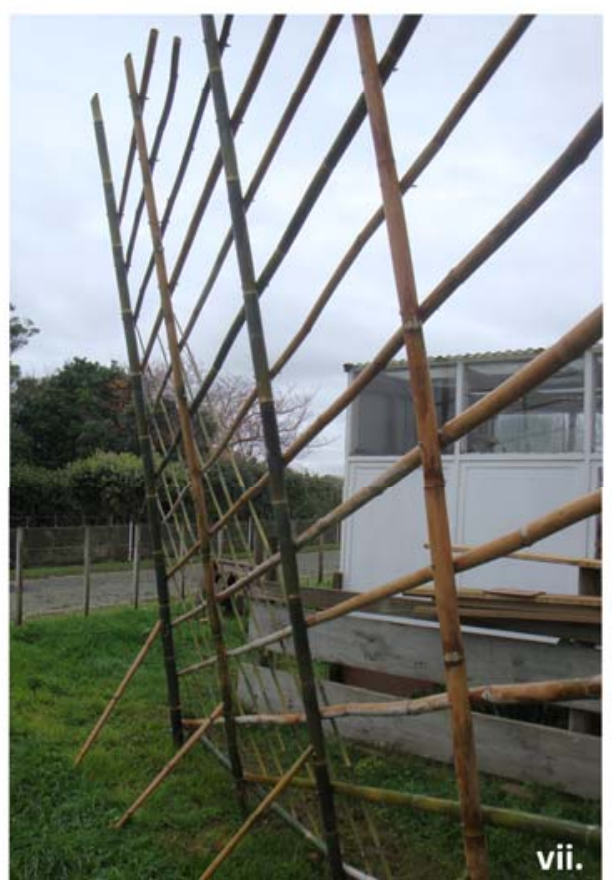

Page | 50

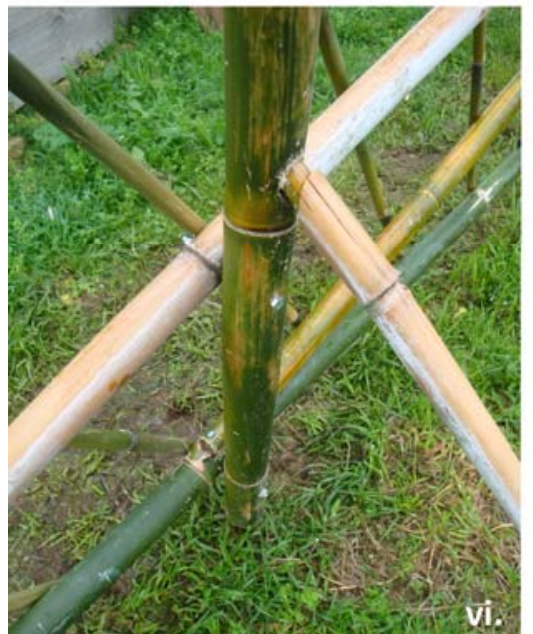


The use of bamboo at different scales allowed the testing of different sized fixings, in particular $50 \mathrm{~mm}$ panhead wood screws which also required pre-drilling, taking significantly longer. The possibility of exterior fixing with bamboo is also difficult as the surface area for fixing a product is significantly smaller.

The green culms had no bending capabilities of benefit over the dried culms. Young culms that were unintentionally used lacked sufficient strength and failed under bending at their nodes and angled supports. Splitting of the culm also increased in young culms with splits extending beyond the nodes. The bending was successful in the horizontal direction, where there was support from the rods that were hammered into the ground; however the vertical curve of the culms recoiled into their original position once the rope was released. A full canopy or arch should be designed with two points of support. 

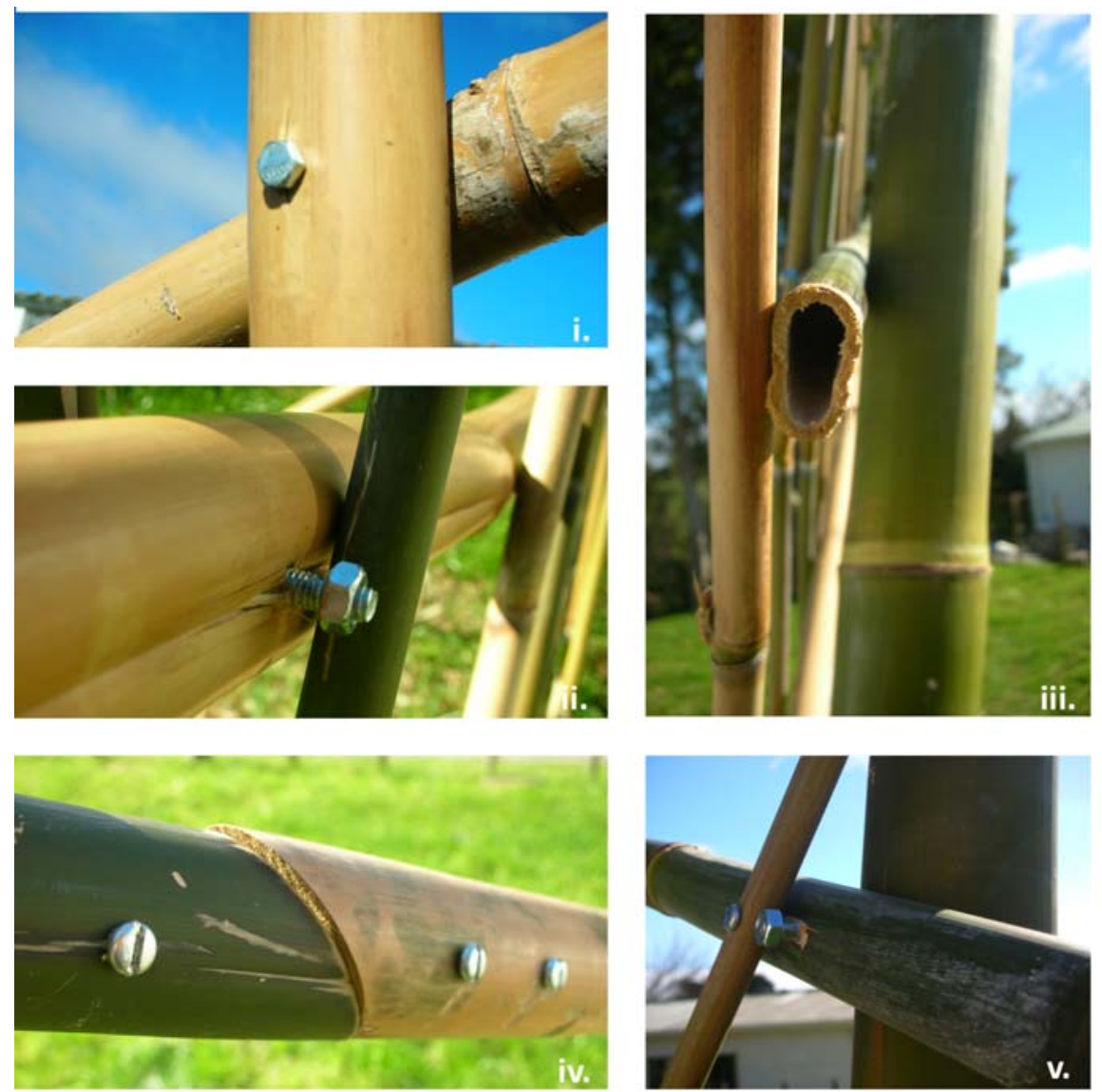

i Connections between dried culms ii Tight fitting bolts now exposed

iii Culm compression splitting occurred

iv Separation between dried and green joints

v Shrinkage between fixing

vi Splitting between bamboo nodes

vii Dried culms were less prone to splitting
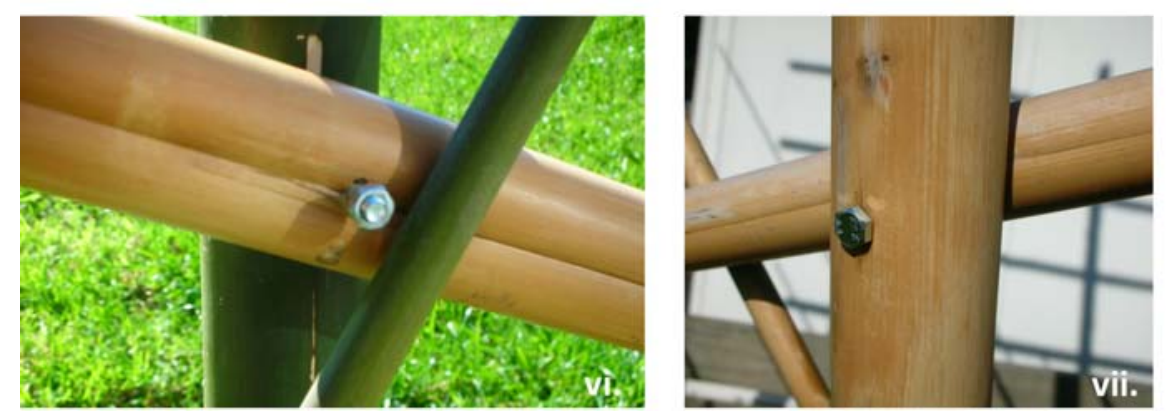

Page | 52 


\subsubsection{Experimentation assessment}

After three months of exposure the bamboo structure was assessed with particular attention being paid toward connections and bamboo deformation. Cracking and/or splitting, and how well the structure held up after exposure to the elements was also examined.

Considerable shrinking had occurred in the green culms resulting in exposed connections and deformation. In areas where pre-drilling and bolted connections occurred, splits between the nodes were evident. An emphasis on designing an adequate connection between bamboo sections without culm penetration needs to be a priority in the design.

Exposure to the elements, and rapid temperature changes had resulted in severe deterioration of the Oldhamii culms, especially in the green culms where the reduction of the moisture in the drying culms had changed their shape and structure. Harvesting at the correct time of 6-7 years of age would result in a much more stable culm.
Exposure to the elements is clearly not desirable, and enclosure is the only option for a suitable design solution. Standard joining techniques need to be refined without culm penetration to ensure splitting does not result. 

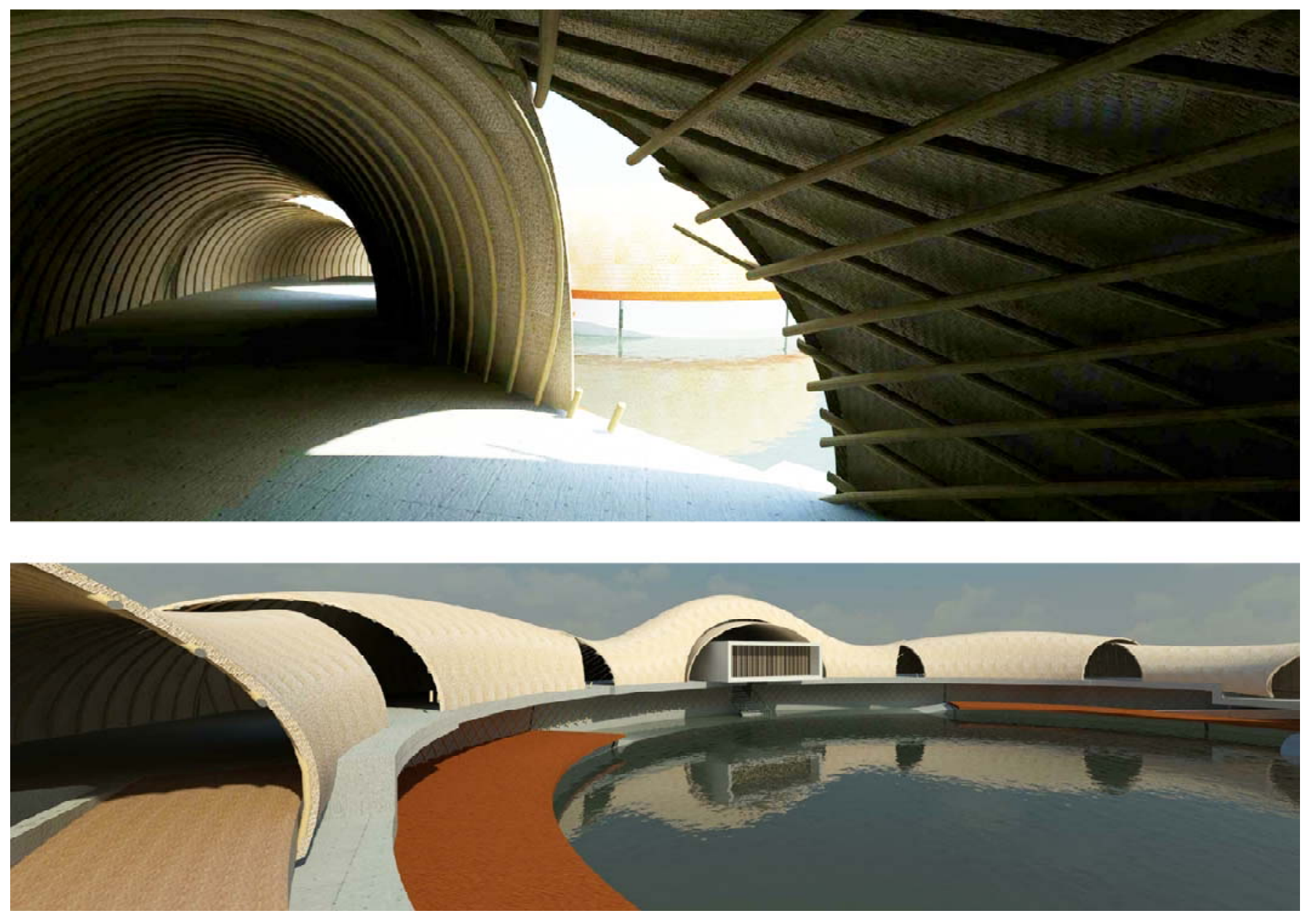

Top Curved forms showing a refined lattice and thin shell structure of BMB Bottom Developed idea showing private enclosed lake pool and surrounding

Page | 54 


\subsubsection{Bamboo development}

The development progressed from the concept and experimentation stage to a design that responded to, and addressed enclosure, while also making use of the culm's curved form. Also important to the concept, is the need to neutralise the unintended indigenous significance. This means shifting away from the cyclic form of the concept, into a more linear driven footprint of the developed bath. The main design feature, the curved canopy is retained, and modified. In both the cyclic and linear forms a contrast between the building and the bamboo canopy is established. The canopy in particular is adapted to have two connection points of support, as explored in the experimentation stage. The lattice structure is also explored in various forms - a single direction, a diamond and a combination of the two.

\subsubsection{Development discussion}

Both Hyams and Mortimer were invited to comment on the design proposals. Their assistance in relation to the use of bamboo in the design was invaluable for understanding how the material would behave in this environment.

In his discussion of the experiments, Hyams recommended drying culms naturally in a ventilated sheltered area, out of the sun before attempting construction. He noted that the drying of bamboo could take up to a year depending on the conditions. A unique aspect of using cut bamboo is that New Zealand beetles do not feed on it, meaning preservation is primarily for moisture. Hyams further stated: “New Zealand beetles don't tend to attack bamboo the same as overseas varieties. There is however a common black fungus that forms on the surface of cut bamboo that can simply be wiped away using detergent." Unlike the life expectancy described in the Preservation of Bamboo report, 

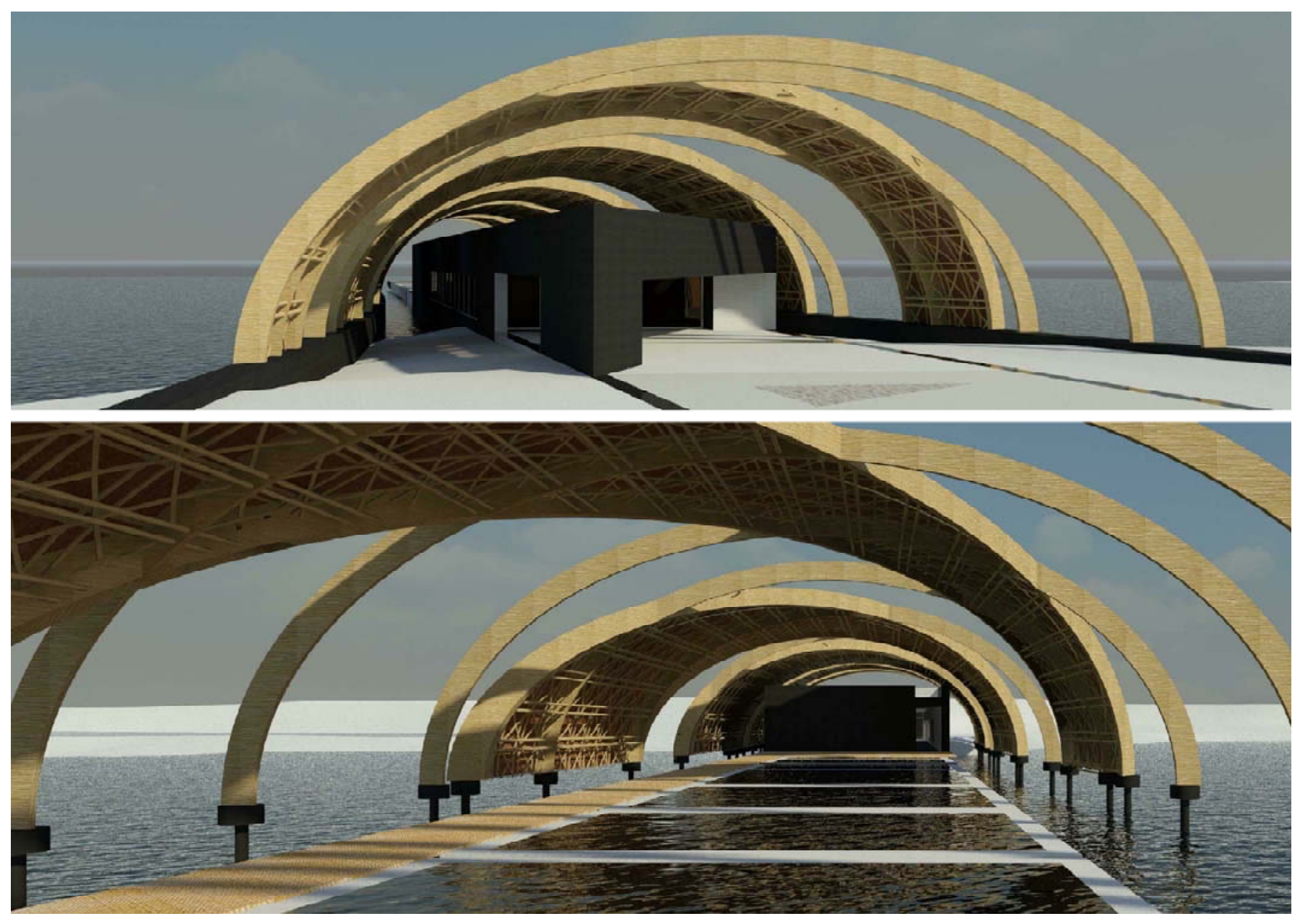

Top Refined design showing integration between natural bamboo,

laminated bamboo and BMB

Bottom View showing canopy over baths

Page | 56 
Hyams suggested that "Exposed bamboo tends to last 20 to 25 years provided it is off the ground and has top protection, about the same as a picket fence, it is quite durable." Because bamboo is unaffected by insects it can be considered an option, with further research, as a potential material for wider applications in New Zealand.

With regard to the design Hyams recommended the use of Moso as it is a structural bamboo that is strong and robust of reasonable thickness, and can be split in halves or quarters. The bamboo needs to resist the effects of capillary absorption by being built above the ground surface, and have significant shelter over the main structure (D. Hyams, personal communication, October 2nd, 2010).

Mortimer, in his discussion of the experimentation, noted that the primary reason that most of the culms failed was because of the age at which they were harvested. The range of culm diameters used in the experiment is also varied. He states, "The diameters of the bamboo available in New Zealand are predominantly larger varieties. A diameter of $60 \mathrm{~mm}$ is a very rare variety. Henonis or Bambusoides usually gets bigger than that, and you've got to go for the smaller ones, which there is less of." The timing of the harvesting, and underestimating the time taken to harvest and process bamboo are very important considerations when dealing with bamboo.

The discussion of the design has challenged Velez' theory; while it is possible to bend bamboo into complex forms, it cannot be done without heating or splitting. Mortimer said:

"From my experience in bending bamboo, it's really hard to, you see all these beautiful curves and everything, but, to actually do it, it's really hard. When you put bamboo under tension like that, it tends to run all over the place. Though you'll never get a catastrophic failure on a weak spot you must watch out for bamboo in bending. Bamboo should be grown into the form or heated and bent into the form. You can't bend it into the form it's too unpredictable."

While bending a full culm naturally is unpredictable, the other method of heating can be used to achieve the curve. The drawback here however, is the removal of the protective layer on 
the bamboo, and the extra labour required to bend the culm.

Mortimer stated:

"A big arch I made had culms of $\varnothing 80 \mathrm{~mm}$. By putting stress on the length and evenly heating using a gas torch, when the bamboo reaches the right temperature it plasticises and bends. Heated bamboo, when it's been really heated, doesn't do very well outside, because you remove one of the protective layers. The lignin plasticises in the fibres at a certain temperature, and you've got to get that to happen without scorching the surface of the culm."

The other option is to create a laminated beam, although there are inherent issues regarding the use of chemicals, "Using bamboo with the minimum amount of processing is great, but it's got limited uses, there's no doubt about it. Once you process it heavily, it becomes a building block, like timber. Like Radiata pine you can build anything out of it." Laminating bamboo also solves the connection issues, "Utilising industrial bamboo removes the problems with joining canes because you can stagger them." By cold forming the bamboo laths with adhesives, much tighter curves can be achieved.

To solve the full culm bending issues, the bamboo must be split into laths. Mortimer said "The beauty of using laths in the construction of the curved shell, is that it's not going to crack because it not a hollow tube anymore, it's just a very flexible lath with all the fibres running up and down." The variety of bamboo used is also important, as runners "have a tendency to be very good for splitting." (M. Mortimer, 2010).

Page | 58 


\subsubsection{Summary}

The design is developed through assistance from interviews and design experimentation to create a design that reflected the flexible, lightweight nature of bamboo (to reflect the connection to the soul). Because bamboo is a unique material with variable properties it requires particular attention to careful design. The experimentation undertaken highlighted issues in maturity and detailing, while the discussion questioned the canopy purpose.

Bamboo selection is the priority when designing with the material. The material must be sufficiently aged and dried before being used in construction. The two dominant timber species in New Zealand are Oldhamii and Moso. Moso in particular is easier for splitting, requires less labour in branch removal and has a clean appearance. Pre-drilling connections required extra labour therefore detailing connections without the need for culm penetration must be a priority. Exposed bamboo is not desirable because of deformation, and increased splitting; therefore an enclosed solution is necessary.

The design developed from predominantly using round bamboo in a solution of curvature into the use of industrial processing to form the curves required. It is possible to achieve the curve in round bamboo using heat bending, however this reduces the natural durability of the culm surface and requires extra labour. Cold forming (Moso) laths into a laminated beam solves the inherent connection, curvature and preservation issues. The use of laths as a secondary structure should also be considered, as tight curves can be achieved and splitting is less likely due to the culm being already split. 


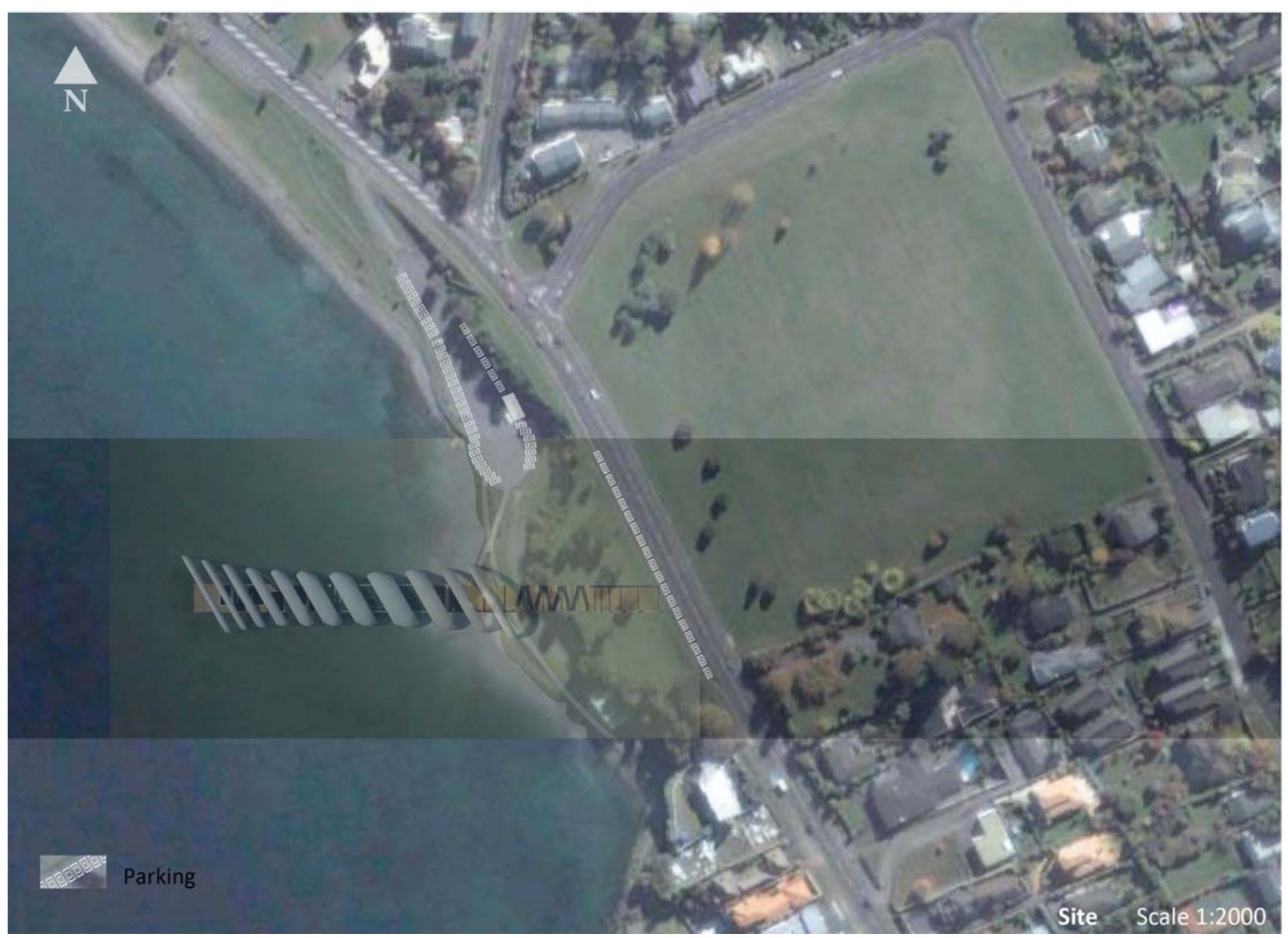

Above Site highlighting parking and relationship to land and water (Google, 2010)

Page | 60 


\subsection{Design solution}

The design solution is discussed in relation to the literature, and the assumptions and directions were chosen in relation to the design. The connection between the bath and the body, the influence of Zumthor's baths on the design, and how bamboo is reflected in the soul and structure of the design were explored.

\subsubsection{Design rationale}

The design concept was drawn from the notion that seismic measurements have a direct relationship to geological movements within the earth's crust, the location at which the geothermal waters originate. This was combined with the idea that ripples in a pool correspond (in appearance) to these seismic readings, forming a larger wave at the point of maximum disturbance to a very shallow wave at the end. The central point then is situated at the centre of the project, around which the rest of the building is based.

This initial concept was reduced as the project developed, however it did provide a simple spatial arrangement for the interior and exterior spaces including the pools and the landscaping. 


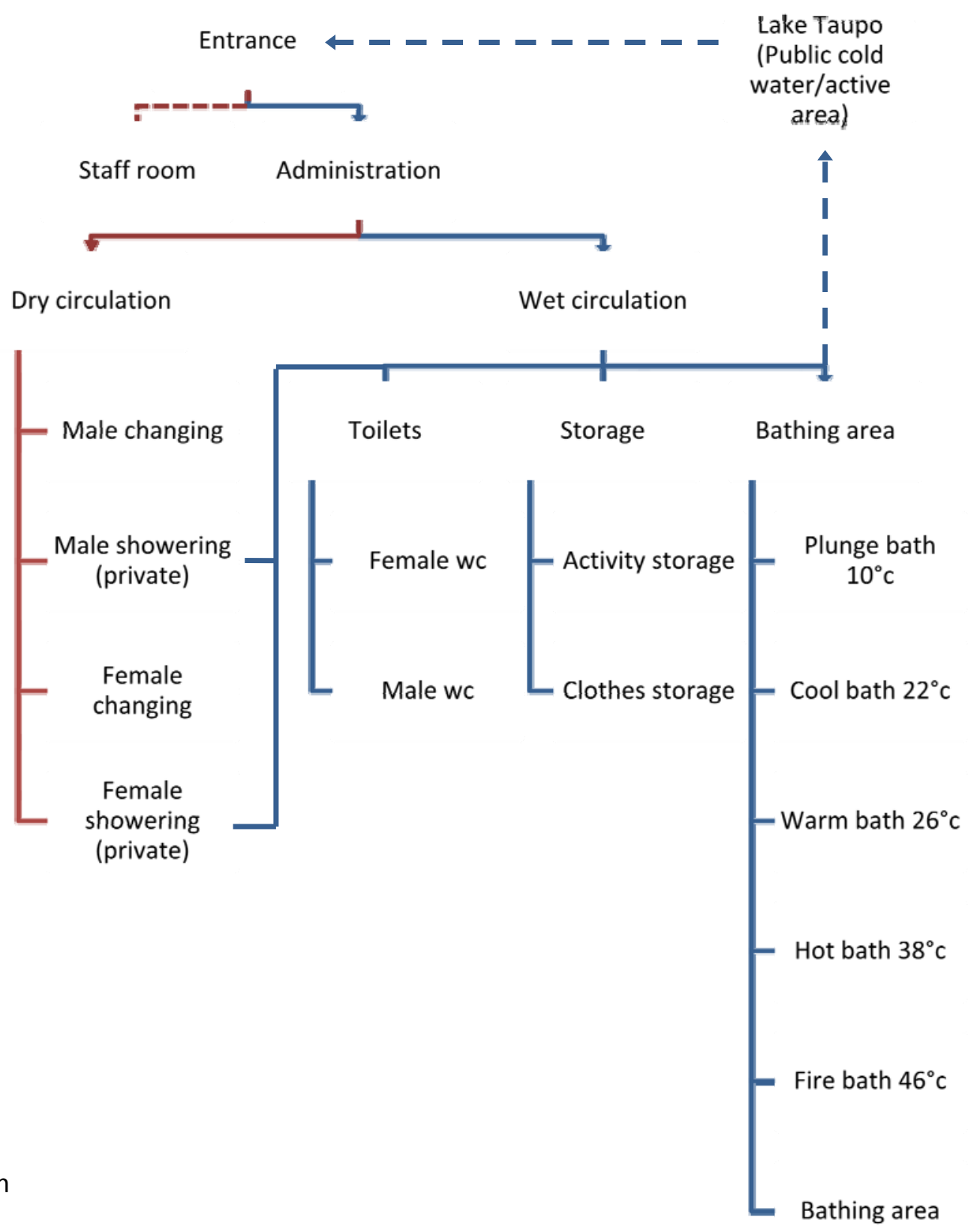

Page | 62 


\subsubsection{Bathing and the body}

The bath design is a combination of relaxation and recreation; both have been incorporated into an outdoor bathing environment. The bath adapts the fundamental principles of alternate temperature bathing from the Greek baths, but also has the added feature of recreational bathing by allowing the user access to the lake, simply by diving from the end of the pier. The temperature fluctuation, from the cold water bath to the hot, encourages people to work their senses by encouraging them to participate in bathing at different temperatures. This expression of bath passage also is a visual cue for the bathers, as the rising vapour from the hot pools at the end of the pier, indicates the hottest pools. This provides a purpose for the bathers to progress to the end of the pier, rewarding their commitment to the progression ritual. The element of entertainment and festivity that the Romans were seeking was not applied to the design, as the focus was predominantly seeking a closer connection between the body and soul; however, some elements of leisure were used. A place for recreational activities (such as board games, snacks and other poolside activities) is required therefore and activity storage will be maintained and operated by a staff member at the entrance to the outdoor pools. The ultimate goal of the space is to reflect on the bath as being a place for relaxing the soul, with the added benefit of social interaction. 


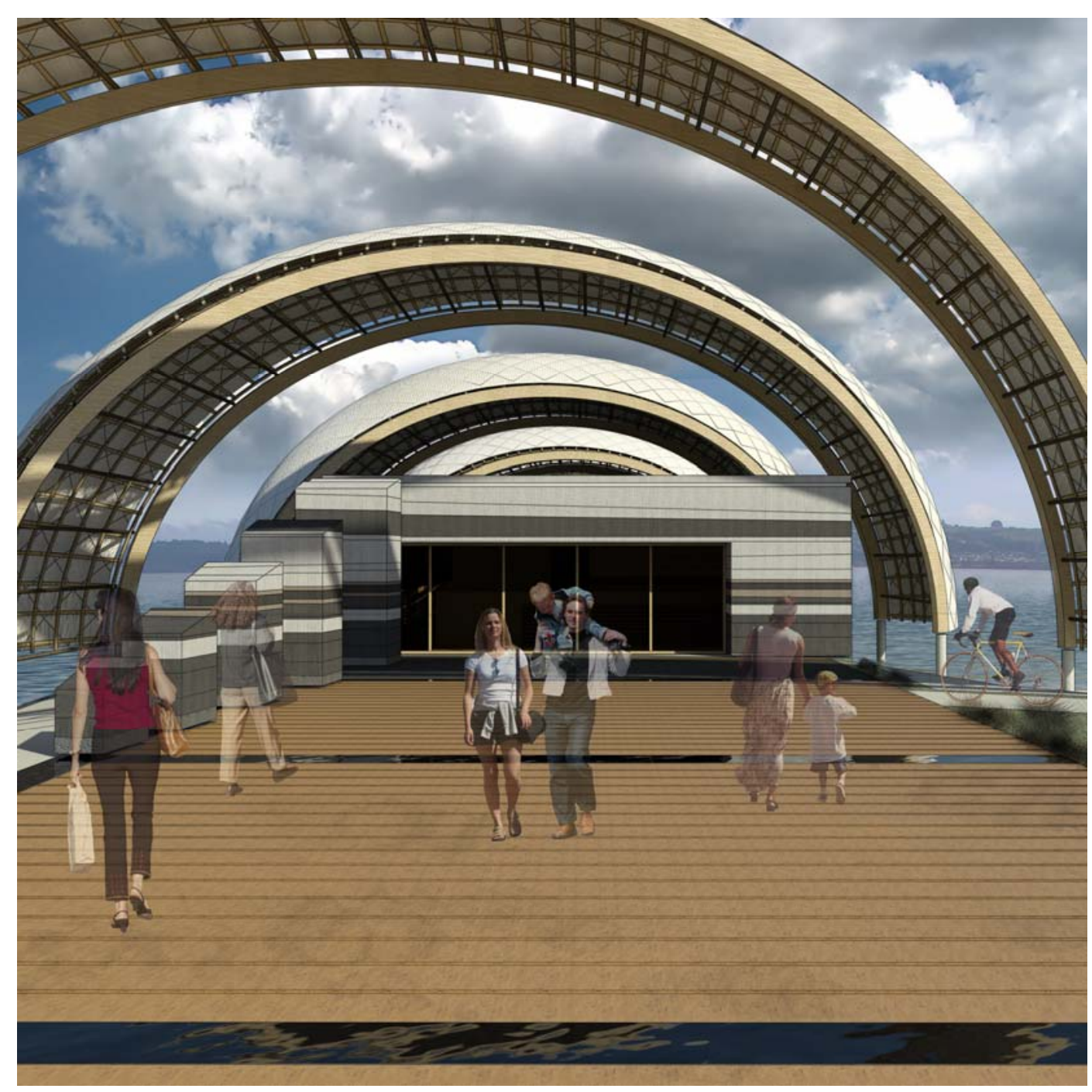

Above Exterior entrance

Page | 64 


\subsubsection{Bath and stone}

Zumthor's baths at Therme Vals highlighted the heavy grounded nature of the baths was reflected in the design were the inspiration for connecting the bath and the body. The varied temperatures of bath were adapted from Zumthor's design, and modified to include depth as a requisite for the different temperature pools, with the cooler pools requiring more activity; their depth was significantly deeper, while the warmer pools were shallower (Longitudinal section).

Unlike Zumthor's curiosity driven passageways, this project reflects on the linear connection routes called bridges. Upon entry patrons are directed through the dry space to the right, into the changing rooms, the left path is used for bathers who swim in the lake and wish to return to the baths. The circulation between the pools is slowed down, by weaving between pools along narrow walkways, slowing the pedestrian movement in the outdoor space.
The architectural style of a heavy grounded mass is reflected in the main body of the building. It is emphasised by the use of oxide impregnated concrete to form 'strata' within the concrete form, reflecting the geological background of the baths. This area contains the reception, changing and toilet facilities, and is a 'bridge' between the town and pool. The bridge is multidirectional, and is seen in the linear passage between the main road and the lake, also between the lake, pool / building and bamboo canopy. The site further connects the linear nature of the project through to the landscaping. 


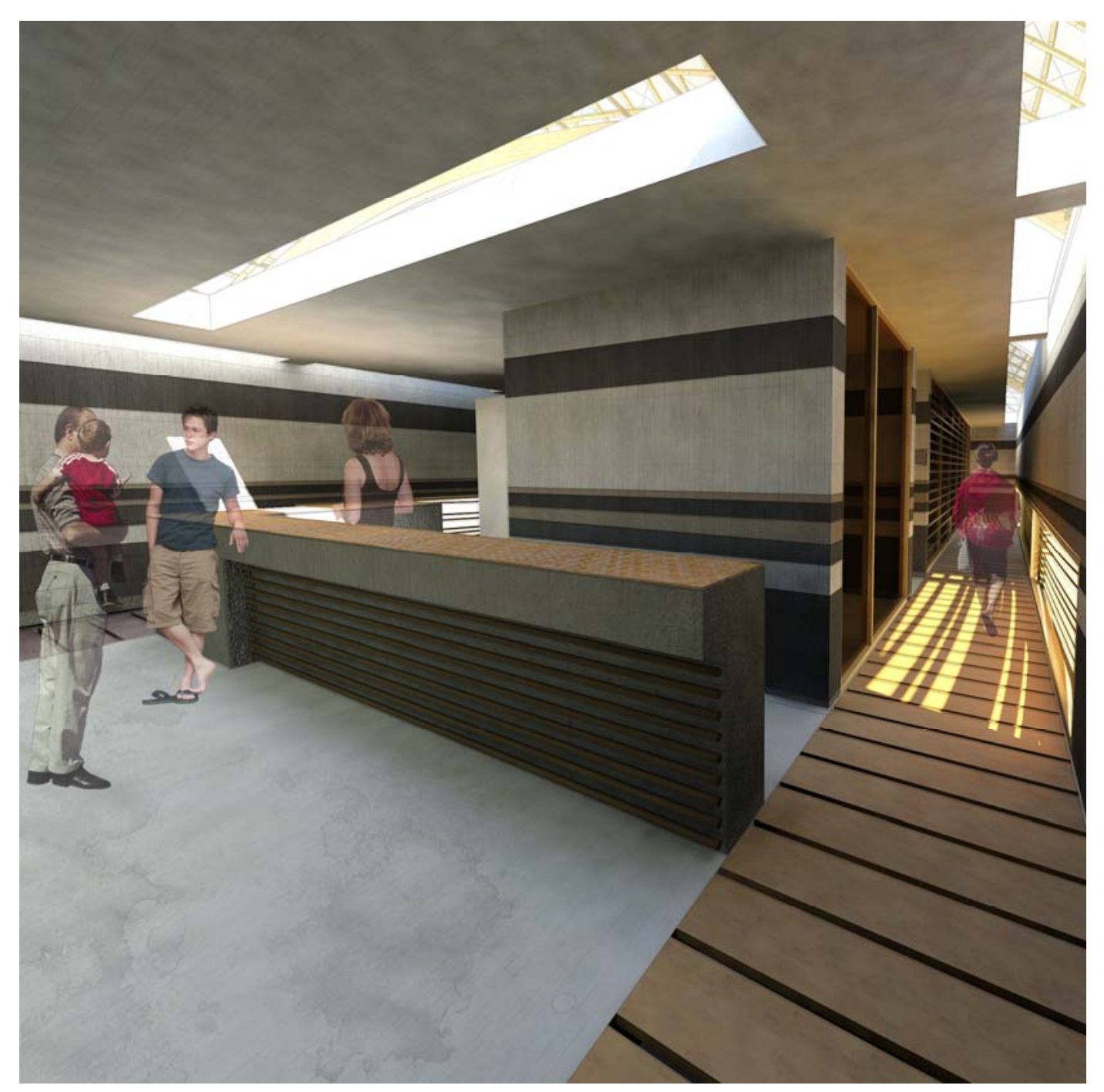

Above Reception area

Page | 66 


\subsubsection{Site and water}

The site is split by the rigid linear passageway from the main road to the lake. This indicates the directional flow of the geothermal waters to the lake, and also the lake's connection to the township. The lake is also is reflected in the landscaping through the use of intentional 'puddles' of water situated throughout the passage, and a 'strata' based planting layout reflecting the different layers through different shrubs and tussocks.

\subsubsection{Bamboo and soul}

The bamboo canopy contrasts with the main bath building on multiple levels: the forms are different, the size and inherent mass differ, and on a plan level the curves also differ. The progression of the pool layout was reflected in the bamboo canopy structure, with canopy and spacing alternating in size and shape. The detached nature between the building and canopy also allows this differentiation between body and soul to be further exaggerated. 


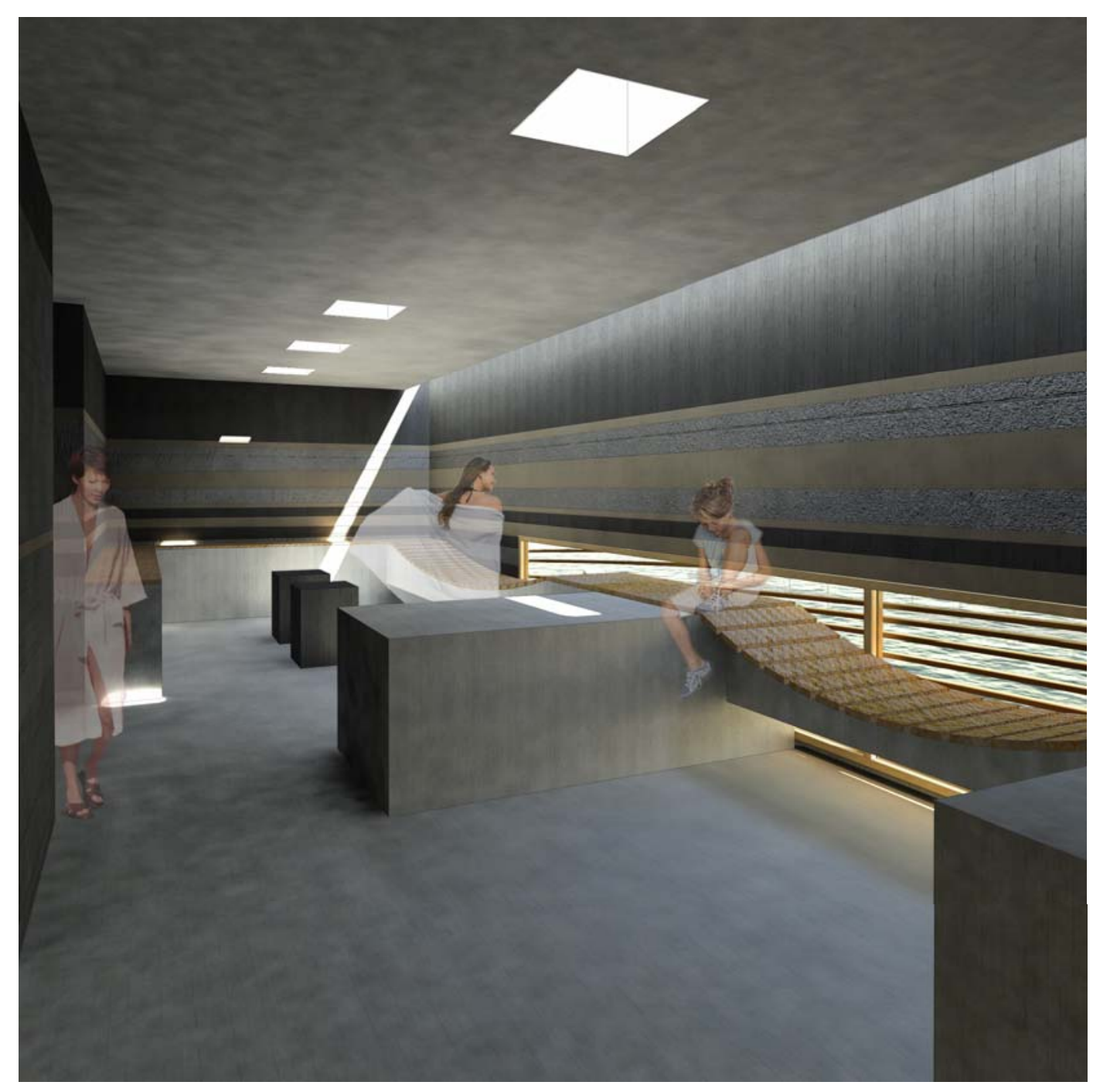

Above Female changing room

Page 68 


\subsubsection{Structure and bamboo}

The bamboo canopy has been adapted throughout the design process to create a design solution that reflected the material's natural flexibility, and light weight. The solution was achieved by using a combination of bamboo and industrial bamboo products to create the canopy. The primary structure is a series of laminated beams running in the North-South direction; this achieves the desired curve and provides the strength needed to support the shell. The beams are supported by a series of Oldhamii struts that are bonded to the beams with mortar infill (a technique adapted from Simon Velez). The lattice is secured to the beams with a series of steel brackets that prevent fixing penetration. The top layer is $\mathrm{BMB}$, which allows a suitable amount of fixing for the BMB panels on top. The canopy achieves a multidirectional flexibility through the use of Moso in the lattice structure, adhering to Velez's discussion on bamboo's potential in the curved form. 


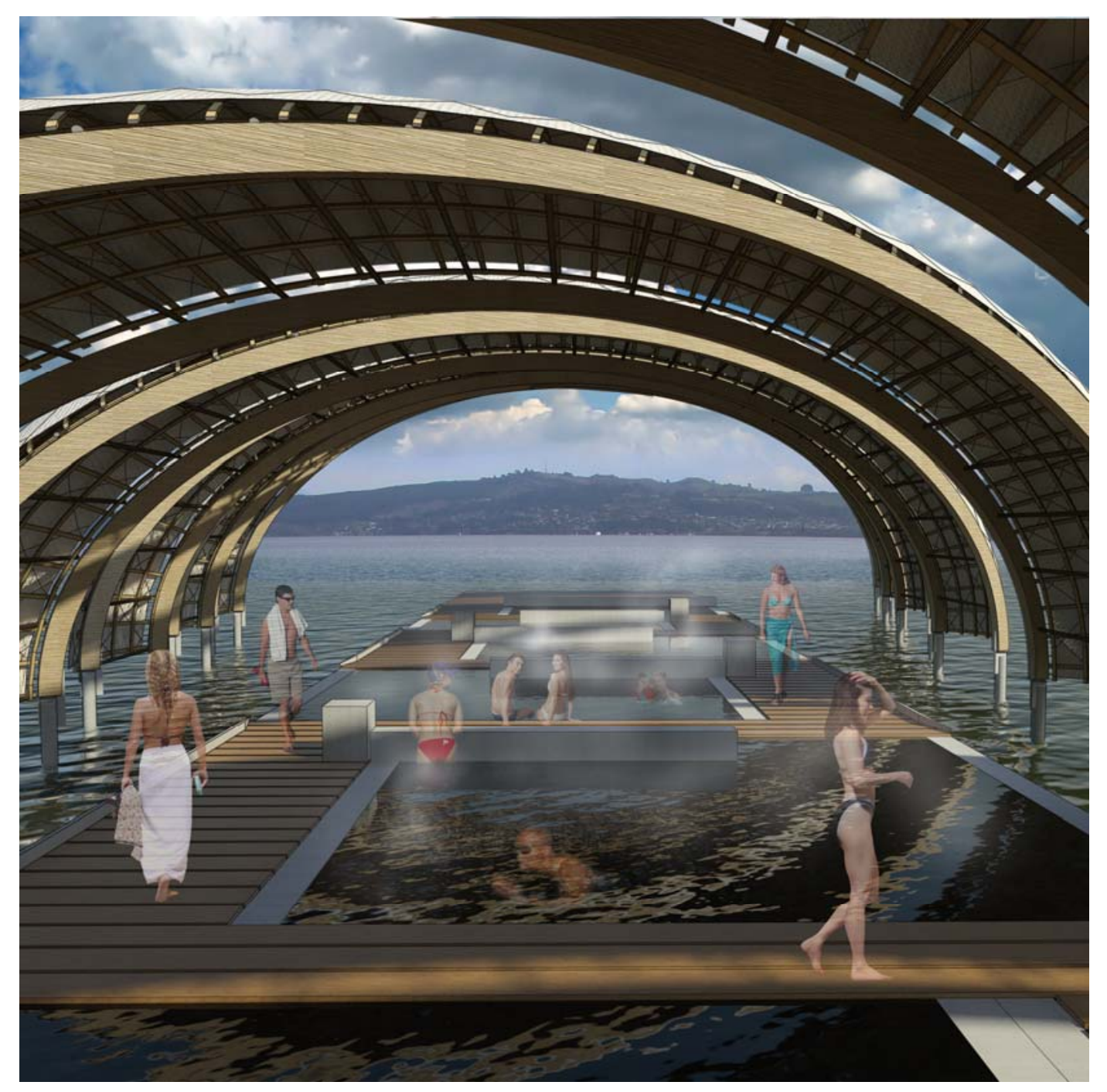

Above Bathing area

Page | 70 


\subsection{Design discussion}

The purpose of the connection between the material contrast of concrete and bamboo, body and soul, heavy and light is reflected clearly in the design. However the functionality of the canopy and the bath structure could be questioned as to its intention for the bathers. The baths relationship to the senses can also be questioned.

The canopy function was questioned as to the relationship between the bath and the semi enclosed nature of the canopy. The canopy serves two functions, firstly privacy from the public and second shelter from the sun. The connection to the surrounding environment is still evident. However the function could perhaps extend into a full overhead shelter in the form of a secondary shelter within the structure.

The use of the roof space on the main building could have further defined the interaction between the bath user and the bamboo canopy. Using the roof as a secondary space for users to relax would enhance the difference between bath and soul. This would exaggerate the contrast between building and canopy further.
The experience of bathing in the baths was considered in relation to the individual depth of each bath and its temperature and layout, progressing from cold to hot. The question arose about how the users would identify the different temperatures of bath? Aside from testing the temperature through touch, and visual cues such as rising vapour, architectural features such as colour clues in each pool might assist in this. This can be challenged by postulating that the rising steam from the hot baths would indicate the temperature change. Minimal architectural expression at the baths also makes for a more direct focus on the bamboo structure. 


\subsection{Conclusion}

The connection between the body and the bath, and the body and the soul can be expressed architecturally through the use of contrasting materiality.

The adaptation of the rituals of bathing into a bath design relating to the process and circulation seen in historical bathing rituals has been further altered into a linear progression from cold to hot, deep to shallow. This simple progression relates well to the fundamental simplicity of Greek and Japanese bathing. Greek and Japanese baths respect the primal purpose of bathing; to cleanse both body and the mind. This is respected in the design by using a simple series of baths that promote visual exploration of the bamboo canopy structure, connecting the body to the soul. The baths' relationship to minerals and topography respect Zumthor's use of the term 'stone water and body,' which is expressed in the use of concrete and large heavy forms. Directly opposing the canopy, this expresses the lightweight and the soul.

The connection between the body and the soul is seen in the connection between the body and the bamboo. Eastern doctrine shares particular connections to the mind or the spirit. Here bamboo is valued for its strength and lightness and the bamboo grove provides a 'sanctuary,' a place of escape, which is reflected in the design. The simplistic bath design allows the user to focus primarily on the material bamboo in the canopy form. Bamboo's flexibility, light-weight and strength are showcased in the different forms of structure. The flexibility is seen in the curved beams and lattice structure, the light-weight in the lattice and $\mathrm{BMB}$, and the strength in the scale of the structure.

Bamboo can be preserved just like any timber-based material can be. However, in contradiction to wider research, New Zealand bamboo has the potential to last up to 25 years without preservation, due to the lack of pests that feed on the introduced grass. Long-term preservation is recommended by the use of a salt-based solution, or industrialised material alternative. Industrial materials have the benefit of preservation, and resolved construction issues when working with the round bamboo culm. The wider potential for $L B L$ and $B M B$ in architecture needs further exploration. The material's use in this project relates to the strength and flexibility of $\mathrm{BMB}$ where it is used as a stretched skin 
membrane across the canopy that complements the lightweight concept. While the LBL form allows the flexibility of bamboo to be explored, its durability is also improved.

The varieties of bamboo available in New Zealand such as Moso, Oldhamii and Latiflorus allow the possibility for further bamboo research and are commonly used in other areas of the world. The material of particular interest is Moso since it requires a less labour intense preparation in contrast to Oldhamii.

The design solution explored the use of both industrial and natural bamboo when combined into a single structure. The experimentation uncovered the priority for sheltering natural bamboo; therefore the need for industrial bamboo was to be used as shelter. The detailing was adapted without penetrating the surface of the bamboo so to not promote the splitting of the (Moso) laths. Careful detailing is essential in bamboo construction to ensure the reduction of culm splitting.

Exploring the connection between body and soul is essential to the development of public bathhouse design. The representation of the body can be seen in the use of heavy, earthly materials and architectural form, while the representation of the soul can also be undertaken materially by using bamboo. Both ideas balance harmoniously with the idea of bathing, in combining body and the soul.

Page | 74 


\section{Reference}

Ashenburg, K. (2007). The Dirt on Clean: An Unsanitized History. New York: North Point Press.

Bonneville, F. D. (1998). The book of the Bath. London: Thames and Hudson Ltd.

Dench, A. (2006). A visitors guide to Taupo and the Central Plateau. Auckland: New Holland Publishers (NZ) Ltd.

Dongchu, H. (1991). The Way of the Virtuous. Beijing, China: New World Press.

Ed. Xiao, Y., Inoue, M. \& Paudel, S.K. (2008). Modern bamboo structures. London: Taylor \& Francis Group.

Farrelly, D. (1984). The Book of Bamboo: A comprehensive guide to this remarkable plant, its uses, and its history. London: Thames and Hudson Ltd.

Ganapathy, P. M., Huan-Ming, Z., Zoolagud, S.S., Turcke, D., \& Espiloy, Z.B (1996). Bamboo Panel Boards - A State of the Art Review. 145 Retrieved from http://www.inbar.int/publication/txt/INBAR Technical Report No12.html

Google. (2010). Google - imagery Retrieved from http://maps.google.co.nz/maps?q=google\%20earth\&oe=utf8\&rls=org.mozilla:en-US:official\&client=firefoxa\&um=1\&ie=UTF-8\&sa=N\&hl=en\&tab=wl

Grilli, P. L., D. (1985). Furo: The Japanese Bath. Tokyo: Kodansha International Ltd.

Hauser, S. (2007). Peter Zumthor therme Vals / essays, Sigrid Hauser, Peter Zumthor ; photographs, Helene Binet ; [translation: Kimi Lum, Catherine Schelbert]. Zurich: Scheidegger \& Spiess.

Velez, S., Vegesack, A,V. \& Kries, M. (2000). Grow your own house : Simón Vélez and bamboo architecture. Weil am Rhein, Germany: Vitra Design Museum : Zeri : C.I.R.E.C.A.
Hidalgo-Lopez, O. (2003). Bamboo gift of the gods. Colombia: D'VINNI Ltd.

Hunter, I. R. (2003). Bamboo resources, uses and trade: the future? Bamboo and Rattan, 2(4).

IPIRTI. (2001). Bamboo Mat Board. Retrieved from http://www.anancy.net/documents/file en/BambooMatBoard. pdf

Keswick, M., \& Jencks, C. (1978). The Chinese Garden: History, Art \& Architecture. Cambridge, Mass: Harvard University Press.

McArthur, M. (2005). The arts of Asia: Materials techniques styles. London: Thames \& Hudson Ltd.

McIntosh, C. (2005). Gardens of the Gods: Myth, Magic and Meaning. London: Tauris \& Co. Ltd.

O'Grady, E. (2009). The Therme Vals / Peter Zumthor. Retrieved 5.11.2010, 2010

Pablo van der Lugt, G. O. (2007). Bamboo Product Commercialization in the European Union An Analysis of Bottlenecks and Opportunities. $100 . \quad$ Retrieved from http://www.inbar.int/publication/txt/INBAR TR 29.htm

Pande, S. K., \& Pandey, S. (2008). Bamboo for the 21st century. International Forrestry Review, 10(2)

Preservation of Bamboo. (2006).

Qisheng, Z., Shenxue, J., \& Yongyu, T. (2003). Industrial Utilization on Bamboo. $212 . \quad$ Retrieved from http://www.inbar.int/publication/txt//tr26/INBAR Technical R eport No26.htm

Sebastian, J. (2010). Norway Pavilion for Shanghai World Expo 2010. Retrieved 18.8, 2010, from http://www.archdaily.com/57891/norway-pavilion-forshanghai-world-expo-2010/

Smith, V. (2007). Clean: A history of personal hygiene. New York: Oxford University Press Inc. 
Spier, S. (2001). Place, authorship and the concrete: three conversations with Peter Zumthor. Arq: Architectural Research Quarterly, 5(1).

Talmadge, E. (2006). Getting Wet. Tokyo: Kodansha International Ltd.

T.D.C. (2010). Taupo District Plan Section 30: Objectives and policies geothermal activity. Taupo District Plan. Retrieved from http://www.taupodc.govt.nz/Documents/Policy/Taupo\%20Distr ict\%20Plan/Current\%20Version/30\%200bjectives\%20and\%20Po licies\%20-\%20Geothermal\%20Activity.pdf

Wang, Z. G., W. (2003). Current status and prospects of new architectural materials from bamboo. Retrieved from http://www.inbar.int/publication/txt/INBAR Working Paper N 047.htm

Zumthor, P. (1996). Thermal Bath at Vals / Peter Zumthor. London: Architectural Association.

Zumthor, P. (1997). Three concepts : Thermal Bath Vals, Art Museum Bregenz, "Topography of Terror" Berlin / Peter Zumthor; Architekturgalerie Luzern, ed. Basel, Boston: Birkhauser Verlag. 


\section{Bibliography}

Amoah, M., Becker, G. \& Nutto, L. (2008). Effects of log export ban policy and dynamics of global tropical wood markets on the growth of timber industry in Ghana. Journal of Forest Economics, 15(2009).

Ashenburg, K. (2007). The Dirt on Clean: An Unsanitized History. New York: North Point Press.

Bansal, A. K., Prasad, T.R.N. (2004). Manufacturing laminates from sympodial bamboos - an Indian experience. Bamboo and Rattan, 3(1), 13-22.

Bonneville, F. D. (1998). The book of the Bath. London: Thames and Hudson Ltd.

Brenner, K. (2008). Bamboo: Mapping Materials. Retrieved $8^{\text {th }}$ April, 2010, from http://www.metrofieldguide.com/portfolio/Bamboo.pdf

Cassandra, A. (1998). Bamboo Architecture and Construction with Oscar Hidalgo. DESIGNER/builder. Retrieved from http://www.networkearth.org/naturalbuilding/bamboo.ht $\mathrm{ml}$

Dench, A. (2006). A visitors guide to Taupo and the Central Plateau. Auckland: New Holland Publishers (NZ) Ltd.

Dongchu, H. (1991). The Way of the Virtuous. Beijing, China: New World Press.

Ed. Xiao, Y., Inoue, M. \& Paudel, S.K. (2008). Modern bamboo structures. London: Taylor \& Francis Group.

Farrelly, D. (1984). The Book of Bamboo: A comprehensive guide to this remarkable plant, its uses, and its history. London: Thames and Hudson Ltd.
Ganapathy, P. M., Huan-Ming, Z., Zoolagud, S.S., Turcke, D., \& Espiloy, Z.B (1996). Bamboo Panel Boards - A State of the Art Review. 145 Retrieved from http://www.inbar.int/publication/txt/INBAR_Technical_Re port_No12.html

Grilli, P. L., D. (1985). Furo: The Japanese Bath. Tokyo: Kodansha International Ltd.

Velez, S., Vegesack, A,V. \& Kries, M. (2000). Grow your own house : Simón Vélez and bamboo architecture. Weil am Rhein, Germany: Vitra Design Museum : Zeri : C.I.R.E.C.A.

Hidalgo-Lopez, O. (2003). Bamboo gift of the gods. Colombia: D'VINNI Ltd.

Hunter, I. R. (2003). Bamboo resources, uses and trade: the future? Bamboo and Rattan, 2(4).

IPIRTI. (2001). Bamboo Mat Board. Retrieved from http://www.anancy.net/documents/file_en/BambooMatB oard.pdf

Jagadeesh, G. (2008). Industrial applications of shock waves Proceedings of the Institution of Mechanical Engineers, Part G: Journal of Aerospace Engineering, 222(5).

Keswick, M., \& Jencks, C. (1978). The Chinese Garden: History, Art \& Architecture. Cambridge, Mass: Harvard University Press.

Knight, D. M. (2007). Going Green with Bamboo. Environmental Design \& Construction, 10(6), 42-44.

Mitchell, C. T., \& Wu, J. (1998). Living Design: The Daoist way of building. United States: McGraw-Hill Companies.

O'Grady, E. (2009). The Therme Vals / Peter Zumthor. Retrieved 5.11.2010, 2010

Pablo van der Lugt, G. O. (2007). Bamboo Product Commercialization in the European Union An Analysis of 
Bottlenecks and Opportunities. 100. Retrieved from http://www.inbar.int/publication/txt/INBAR_TR_29.htm

Pande, S. K., \& Pandey, S. (2008). Bamboo for the 21st century. International Forrestry Review, 10(2).

Qisheng, Z., Shenxue, J., \& Yongyu, T. (2003). Industrial Utilization on Bamboo. 212. Retrieved from http://www.inbar.int/publication/txt//tr26/INBAR_Technic al_Report_No26.htm

Roach, M. (1996). Bamboo Solution. Discover, 17(6).

Rowthorn, C., Ashburne, J., Atkinson, D., Bender, A., Benson, S. \& McLachlan, C. (2003). Lonely Planet: Japan Lonely Planet Publications Pty Ltd.

Sebastian, J. (2010). Norway Pavilion for Shanghai World Expo $2010 . \quad$ Retrieved 18.8, 2010, from http://www.archdaily.com/57891/norway-pavilion-forshanghai-world-expo-2010/

Smith, V. (2007). Clean: A history of personal hygiene. New York: Oxford University Press Inc.

Spier, S. (2001). Place, authorship and the concrete: three conversations with Peter Zumthor. Arq: Architectural Research Quarterly, 5(1).

Steinman, A. (2010). The Four Gentleman or The Four Seasons: (Bamboo, Chrysanthemum, Plum Blossom, Orchid). Retrieved 18.6.2010, from http://www.annesteinman.com/Four Gentlemen.htm

Sulastiningsih, I. M., \& Nurwati. (2009). Physical and Mechanical Properties of Laminated Bamboo Board. Journal of Tropical Forest Science, 21(3), 246-251.

Sunley, J., \& Bedding, B. (1985). Timber in Construction. London: Batsford/TRADA.
Talmadge, E. (2006). Getting Wet. Tokyo: Kodansha International Ltd.

Tsumura, A. (1992). The Japanese Furo Trendy Bath Life. Tokyo: Japan Publications.

Van Der Lugt, P., Van Den Dobbelsteen, A., \& Abrahams, R. (2003). Bamboo as a building material alternative for Western Europe? A study of the environmental performance, costs and bottlenecks of the use of bamboo (products) in Western Europe. Bamboo and Rattan, 2(3).

Vantomme, P., Braulin, N, Chioetto, V \& Liese, W. (2003). Public constructions made with bamboo: lessons learnt from the 'Vergiate bamboo pavilion' in Northern Italy. Bamboo and Rattan, 2(4), 369-380.

Wang, Z. G., W. (2003). Current status and prospects of new architectural materials from bamboo. Retrieved from http://www.inbar.int/publication/txt/INBAR_Working_Pap er No47.htm

Wang, Z. G., G. (2003). Laminated Panel Manufacture of Two Kinds of Bamboo for Architecture Material and Property Comparison. Retrieved from http://www.inbar.int/publication/txt/INBAR_Working_Pap er_No48.htm

Whittaker, P. (2005). Hardy Bamboos: Taming the Dragon. United States, Portland: Timber Press, Inc.

Xu, Q. S. Z. B. (2001). Transfer of Technology Model: Bamboo Floor Manufacturing Unit

Zumthor, P. (1997). Three concepts: Thermal Bath Vals, Art Museum Bregenz, "Topography of Terror" Berlin / Peter Zumthor ; Architekturgalerie Luzern, ed. Basel, Boston: Birkhauser Verlag. 

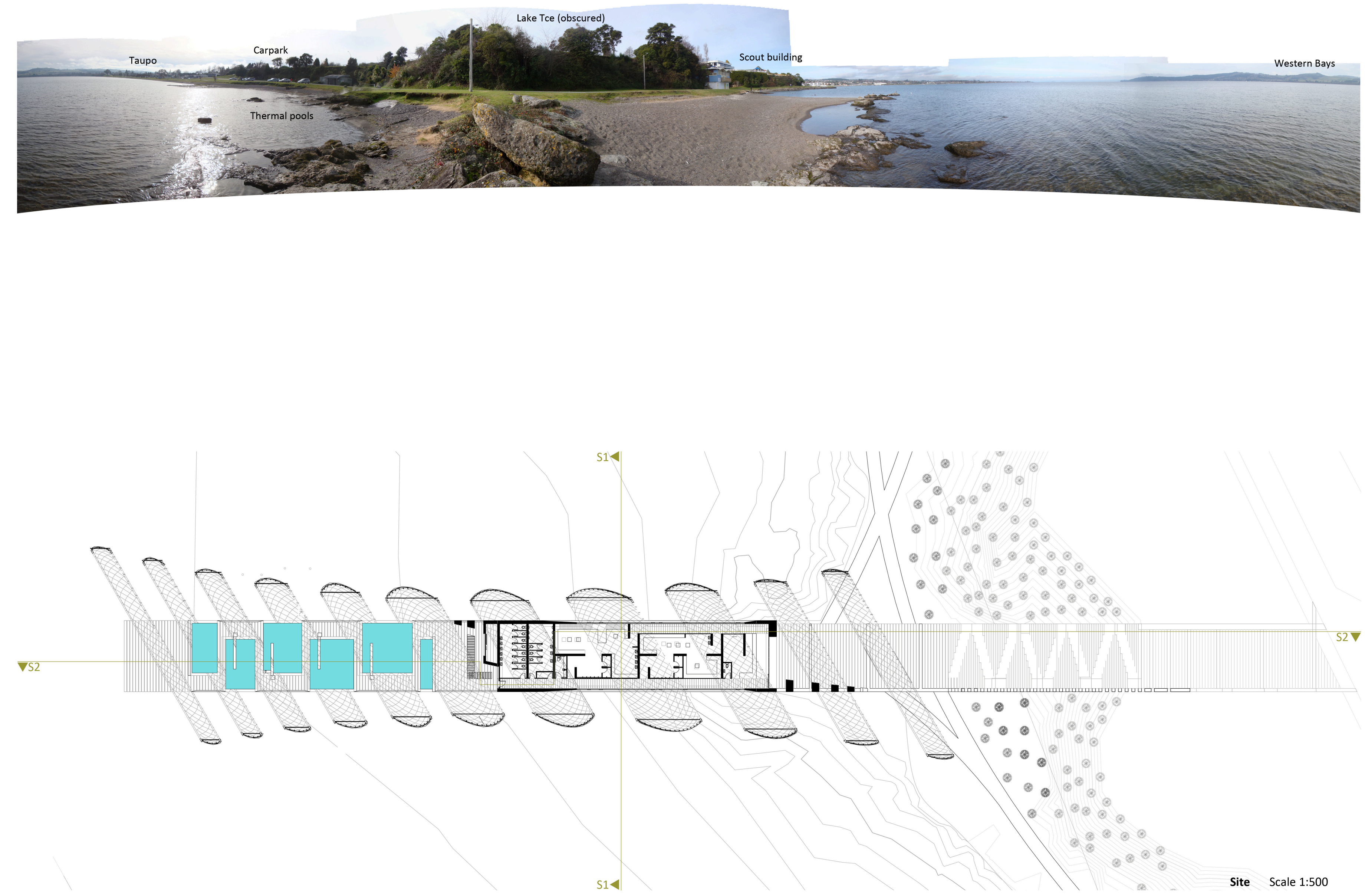

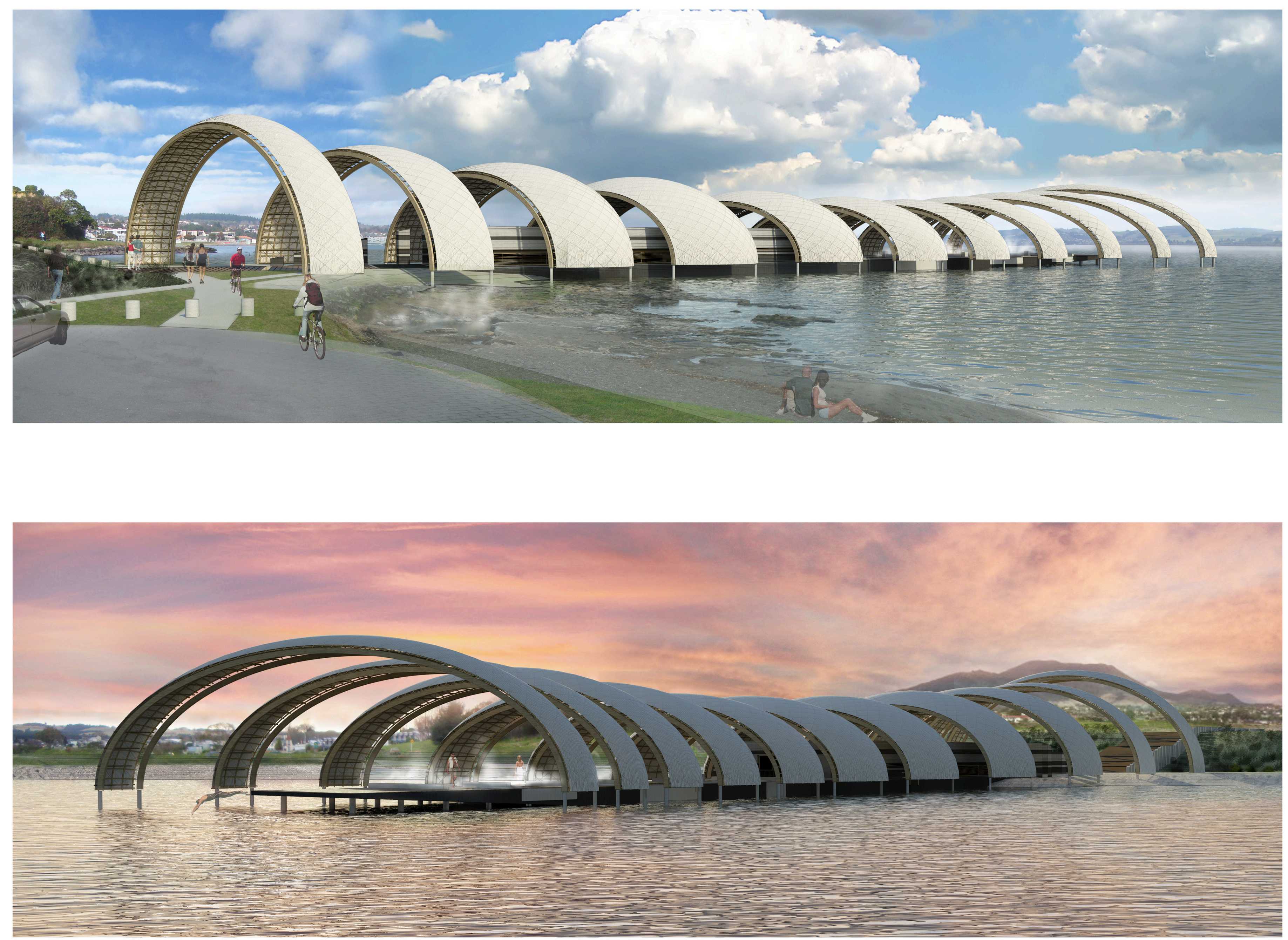

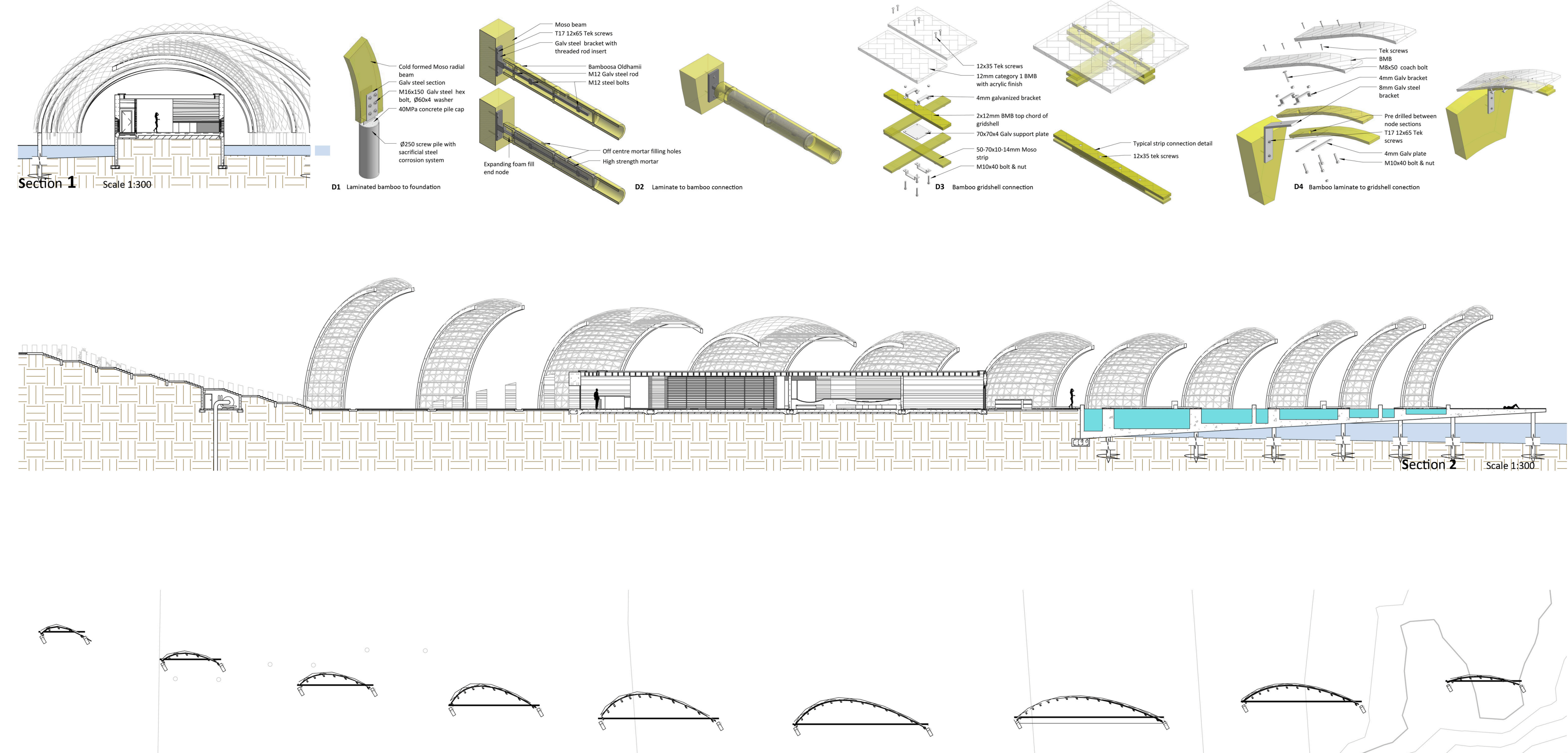

1.Bathing Area
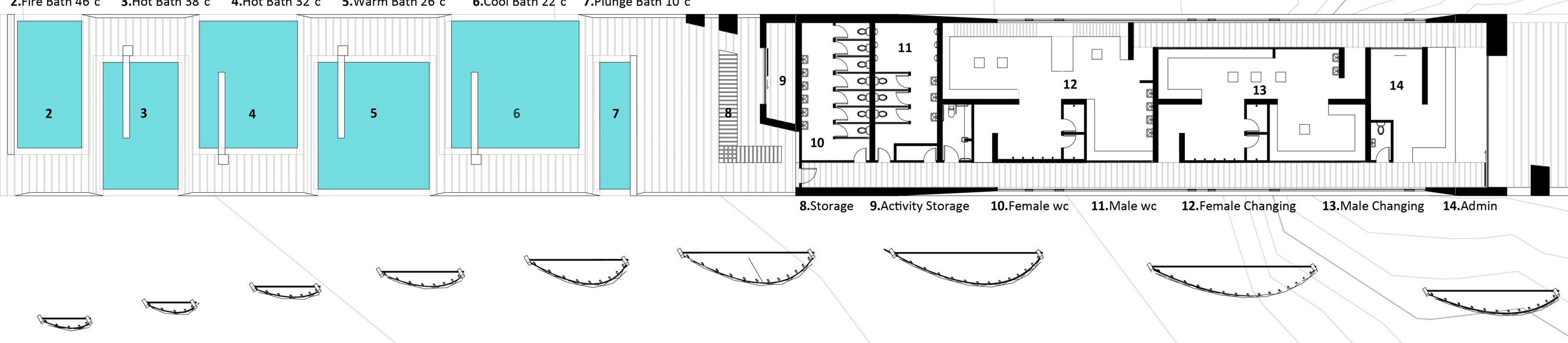